\title{
Asymptotic velocity for four celestial bodies
}

\author{
Andreas Knauf*
}

June 21, 2018

\begin{abstract}
Asymptotic velocity is defined as the Cesàro limit of velocity. As such, its existence has been proven for bounded interaction potentials. This is known to be wrong in celestial mechanics with four or more bodies.

Here we show for a class of pair potentials including the homogeneous ones of degree $-\alpha$ for $\alpha \in(0,2)$, that asymptotic velocities exist for up to four bodies, dimension three or larger, for any energy and almost all initial conditions on the energy surface.
\end{abstract}

\section{Introduction}

In classical scattering theory one considers the motion of $n$ particles of masses $m_{i}>0$ and positions $q_{i}$ in $d$ spatial dimensions, generated by the Hamiltonian function

$$
H(p, q):=K(p)+V(q) \quad, \quad \text { with } V(q):=\sum_{1 \leq i<j \leq n} V_{i, j}\left(q_{i}-q_{j}\right),
$$

and kinetic energy $K(p):=\sum_{i=1}^{n} \frac{\left\|p_{i}\right\|^{2}}{2 m_{i}}$. The potential $V$ is assumed to be twice continuously differentiable and long-ranged, that is, for some $\varepsilon>0, I>0$ and in multi-index notation with $\beta \in \mathbb{N}_{0}^{d}$

$$
\left|\partial_{\beta} V_{i, j}(q)\right| \leq I\|q\|^{-\varepsilon-|\beta|} \quad(|\beta| \leq 2,\|q\| \in[1, \infty), 1 \leq i<j \leq n) .
$$

For long-ranged $V_{i, j} \in C^{2}\left(\mathbb{R}^{d}, \mathbb{R}\right)$ and phase space $P:=T^{*} \mathbb{R}^{d n}$, the Hamiltonian flow exists for all times (the escape times equal $T^{ \pm} \equiv \pm \infty$ ). For all initial conditions the existence of the asymptotic velocities, that is, the Cesàro limits

$$
\bar{v}^{ \pm}\left(x_{0}\right):=\lim _{t \rightarrow T^{ \pm}\left(x_{0}\right)} \frac{q\left(t, x_{0}\right)}{t} \in \mathbb{R}^{d n} \quad\left(x_{0} \in P\right)
$$

*Department of Mathematics, Friedrich-Alexander-University Erlangen-Nürnberg, Cauerstr. 11, D-91058 Erlangen, Germany, knauf@math.fau.de 
of velocity $v$ (with momenta $p_{i}=m_{i} v_{i}$ ), was proven in [De, Theorem 3.1]. So in the infinite past and future the particles cluster, i.e. form set partitions of $N:=\{1, \ldots, n\}$ with equivalence relations given by $i \sim j$ if $\bar{v}_{i}^{ \pm}\left(x_{0}\right)=\bar{v}_{j}^{ \pm}\left(x_{0}\right)$.

Existence of asymptotic velocities is a fundamental result in classical scattering theory, whose proof is quite involved (see also [Kn, Chapter 12.6]). As the $\bar{v}^{ \pm}$are constants of the motion, they present a weak form of integrability. In fact, in the non-empty open set of $x_{0}$ leading to a trivial cluster partition (all $\bar{v}^{+}\left(x_{0}\right)$ being different), $\bar{v}^{+}$is smooth if $(1.2)$ is valid for all multiindices $\beta \in \mathbb{N}_{0}^{n d}$. However, in the presence of nontrivial clusters, one would need more constants of the motion or other asymptotic information [MS1, Sa] to have the so-called asymptotic completeness, see [De].

For the unbounded potentials considered here, the question of classical asymptotic completeness is even more involved. In the case of celestial mechanics, that is (in units where the gravitational constant equals one), $V_{i, j}(q)=-\frac{m_{i} m_{j}}{\|q\|}$, there are collisions for $n \geq 2$, and non-collision singularities for $n \geq 4$, see [Xi, Ge, Xu] and Section 2. Moreover, as shown in [SX] for $d=1$ and $n=4$ there are initial conditions which (after regularization of binary collisions) do not lead to singularities but for which $\lim _{t \rightarrow+\infty}\left\|q\left(t, x_{0}\right)\right\| / t=\infty$, so that asymptotic velocity (1.3) does not exist (see also [SD]).

Conversely, for $n<4$ celestial bodies asymptotic velocities exist for all initial conditions. This follows from von Zeipel's Theorem and the fact that $j^{+}(x)<\infty$ for $n \leq 3$, see Theorem 2.6.

1.1 Definition The potential $V$ in (1.1) is admissible if it is long-ranged, central $\left(V_{i, j}(q)=W_{i, j}(\|q\|)\right.$ for $\left.q \in \mathbb{R}^{d} \backslash\{0\}\right)$, and for some $\alpha \in(0,2)$ and $I>0$

$$
\left|\partial_{\beta} V_{i, j}(q)\right| \leq I\|q\|^{-\alpha-|\beta|} \quad(|\beta| \leq 2,\|q\| \in(0,1], 1 \leq i<j \leq n) .
$$

1.2 Remark (The constant $I$ ) By taking the maximum, we will use the same constant $I$ in (1.2) and (1.4). To gain more flexibility, by further increasing $I$, we assume that (1.4) is valid even for $\|q\| \leq 2\left(\sum_{i=1}^{n} m_{i}\right) / \min _{i} m_{i}$.

\subsection{Example (Homogeneous potentials are admissible)}

If the pair potentials are homogeneous, that is, for $\alpha_{i, j} \in(0,2)$ and $I_{i, j} \in \mathbb{R}$

$$
V_{i, j}(q)=I_{i, j}\|q\|^{-\alpha_{i, j}} \quad\left(q \in \mathbb{R}^{d} \backslash\{0\}, 1 \leq i<j \leq n\right),
$$

then $V$ is admissible. This includes gravitational and electrostatic interactions. $\diamond$

Our main result is the following.

Theorem For $n=4$ bodies, $d \geq 3$ dimensions, and an admissible potential $V$ 
the sets NAV $:=\left\{x \in P \mid \bar{v}^{+}(x)\right.$ does not exist $\}$ of no asymptotic velocity and

$$
\mathrm{NAV}_{E}:=\mathrm{NAV} \cap \Sigma_{E} \quad \text { with } \quad \Sigma_{E}:=H^{-1}(E) \quad(E \in \mathbb{R})
$$

are Borel sets, with $\mathrm{NAV}_{E} \subseteq \Sigma_{E}$ being of Liouville measure $\sigma_{E}\left(\mathrm{NAV}_{E}\right)=0$.

So in this case the asymptotic velocities (1.3) exist almost everywhere. Theorem 2.8 of Section 2 analyzes the set NAV for $n \in \mathbb{N}$ particles in arbitrary dimension $d$, interacting via long-ranged potentials.

\section{Content}

In Section 2 we will introduce some notation and present the main ideas of the proof. A nondeterministic kinematical model for the asymptotics of those orbits, whose asymptotic velocity does not exist, is exhibited in Section 3. That the dynamics is in fact well described by that model, will be shown in Section 4 . The proof of our main result is based on a Poincaré section method devised in [FK1]. The corresponding estimates form the content of the final Section 5.

Acknowledgements I am grateful to Stefan Fleischer for many conversations about the subject of non-collision singularities. The critical remarks of the anonymous referee were very helpful for improving the text.

\section{Almost sure existence of asymptotic velocity}

\subsection{Some general notation}

In general, for a $C^{1}$-vector field $X: P \rightarrow T P$ on a manifold $P$, we denote its maximal flow by $\Phi$. Then

$$
\Phi \in C^{1}(D, P) \text { with domain } D:=\left\{(t, x) \in \mathbb{R} \times P \mid t \in\left(T^{-}(x), T^{+}(x)\right)\right\}
$$

and its escape times $T \equiv T^{+}: P \rightarrow(0,+\infty]$ and $T^{-}: P \rightarrow[-\infty, 0) . T$ is lower semicontinuous in the obvious topology of $(0, \infty]:=(0, \infty) \cup\{\infty\}$.

We mark the values of a phase space variable $V \in C^{1}\left(P, \mathbb{R}^{d}\right)$ along the flow line $t \mapsto \Phi_{t}(x)$ by a tilde:

$$
\widetilde{V}(t):=V \circ \Phi_{t}(x) .
$$

For one-sided limits we use the notations

$$
\widetilde{V}\left(t^{+}\right):=\lim _{s \searrow t} \tilde{V}(s) \text { and } \tilde{V}\left(t^{-}\right):=\lim _{s \nearrow t} \tilde{V}(s) .
$$

Here we consider $n$ particles of masses $m_{i}>0$ in the configuration spaces $M_{i}:=$ $\mathbb{R}^{d}$. On the joint configuration space $M:=\bigoplus_{i=1}^{n} M_{i}$ of all particles we use the inner product $\langle\cdot, \cdot\rangle_{\mathcal{M}}$ generated by the mass matrix $\mathcal{M}:=\operatorname{diag}\left(m_{1}, \ldots, m_{n}\right) \otimes \mathbb{1}_{d}$,

$$
\langle\cdot, \cdot\rangle_{A}: M \times M \rightarrow \mathbb{R} \quad, \quad\left\langle q, q^{\prime}\right\rangle_{A}:=\left\langle q, A q^{\prime}\right\rangle,
$$


denoting the bilinear form for the matrix $A \in \operatorname{Mat}(M, \mathbb{R})$, with the canonical inner product $\langle\cdot, \cdot\rangle$. We set $\|q\|_{A}:=\langle q, q\rangle_{A}^{1 / 2}$.

The collision set in the configuration space is given by

$$
\Delta:=\left\{q \in M \mid q_{i}=q_{j} \text { for some } i \neq j \in N\right\} .
$$

We consider potentials $V \in C^{2}(\widehat{M}, \mathbb{R})$ on the non-collision configuration space $\widehat{M}:=M \backslash \Delta$. The Hamiltonian function $H \in C^{2}(P, \mathbb{R})$ on the phase space $P:=T^{*} \widehat{M}$ is given by (1.1), with pair potentials $V_{i, j}$.

With the Euclidean gradient $\nabla$ on $\mathbb{R}^{d}$ the Hamiltonian equations of (1.1) are

$$
\dot{p}_{i}=\sum_{j \in N \backslash\{i\}} \nabla V_{i, j}\left(q_{j}-q_{i}\right) \quad, \quad \dot{q}_{i}=\frac{p_{i}}{m_{i}} \quad(i \in N) .
$$

Using the natural symplectic form $\omega_{0}$ on the cotangent bundle $P$, we write these as $\dot{x}=X_{H}(x)$ for the Hamiltonian vector field $X_{H}$ defined by $\mathbf{i}_{X_{H}} \omega_{0}=d H$.

Since $H \circ \mathcal{R}=H$ for the involution $\mathcal{R}: P \rightarrow P,(p, q) \mapsto(-p, q)$, the maximal flow $\Phi \in C^{1}(D, P)$ is reversible: $\mathcal{R} \circ \Phi_{t} \circ \mathcal{R}=\Phi_{-t}$. In particular $T^{-}(p, q)=-T^{+}(-p, q)$. $\Phi$ restricts to the energy surfaces $\Sigma_{E}:=H^{-1}(E)$. We write $(p(t, x), q(t, x)):=\Phi(t, x)$ for the momenta resp. positions at time $t$ starting at $x \in P$, and given an initial condition $x$, we even write $(\tilde{p}(t), \tilde{q}(t))$.

2.1 Definition The set of phase space points experiencing a singularity is

$$
\text { Sing }:=\{x \in P \mid T(x)<\infty\},
$$

whereas its subsets experiencing a (non-) collision singularity are

$$
\text { Coll }:=\left\{x \in \text { Sing } \mid \lim _{t \succ T(x)} q(t, x) \text { exists }\right\} \quad \text { and } \quad \mathrm{NC}:=\text { Sing } \backslash \text { Coll . }
$$

The corresponding orbits in Coll resp. NC are called (non-) collision orbits.

Sing $\cap \mathrm{NAV}=\mathrm{NC}$, and Sing, Coll and NC are Borel subsets of $P$.

For arbitrary $n \in \mathbb{N}, d \geq 2$ and a large class of pair interactions including the homogeneous ones (see Saari [Sa1]) the set of initial conditions leading to collisions are of Liouville measure zero for all total energies $E$, see [FK2]. Strongly based on finiteness of the escape time, the same result concerning non-collision singularities was proven for $n=4$ by Saari in [Sa2] and by Fleischer in [Fl].

To show almost sure existence of asymptotic velocities, we have to take into account the case of solutions of the initial value problem that exist for all times, but where (1.3) does not exist. So we have to argue differently from [Sa2, FI]. 


\subsection{Cluster decompositions}

This section can be skipped, as we recall the notations introduced here in later sections. We introduce standard notions for the set partitions of $N=\{1, \ldots, n\}$ :

\subsection{Definition}

- A set partition or cluster decomposition of $N$ is a set $\mathcal{C}:=\left\{C_{1}, \ldots, C_{k}\right\}$ of blocks or clusters $\emptyset \neq C_{\ell} \subseteq N$ such that

$$
\bigcup_{\ell=1}^{k} C_{\ell}=N \quad \text { and } \quad C_{\ell} \cap C_{m}=\emptyset \text { for } \ell \neq m .
$$

We denote by $\sim_{\mathcal{C}}$ (or $\sim$, if there is no ambiguity) the equivalence relation on $N$ induced by $\mathcal{C}$; the corresponding equivalence classes are denoted by $[\cdot]_{\mathcal{C}}$.

- The lattice of partitions $\mathcal{P}(N)$ is the set of cluster decompositions $\mathcal{C}$ of $N$, partially ordered by refinement, i.e.,

$$
\mathcal{C}=\left\{C_{1}, \ldots, C_{k}\right\} \preccurlyeq\left\{D_{1}, \ldots, D_{\ell}\right\}=\mathcal{D},
$$

if $C_{m} \subseteq D_{\pi(m)}$ for an appropriate surjection $\pi:\{1, \ldots, k\} \rightarrow\{1, \ldots, \ell\}$.

In this case, $\mathcal{C}$ is called finer than $\mathcal{D}$ and $\mathcal{D}$ coarser than $\mathcal{C}$.

The unique finest and coarsest elements of $\mathcal{P}(N)$ are

$$
\mathcal{C}_{\wedge}:=\{\{1\}, \ldots,\{n\}\} \quad \text { and } \quad \mathcal{C}_{\vee}:=\{N\}=\{\{1, \ldots, n\}\},
$$

respectively. Two clusters $\mathcal{C}, \mathcal{D} \in \mathcal{P}(N)$ are comparable if $\mathcal{C} \preceq \mathcal{D}$ or $\mathcal{C} \succeq \mathcal{D}$.

- The rank of $\mathcal{C} \in \mathcal{P}(N)$ is the number $|\mathcal{C}|$ of its blocks, and

$$
\mathcal{P}_{k}(N):=\{\mathcal{C} \in \mathcal{P}(N)|| \mathcal{C} \mid=k\} \quad(k \in N) .
$$

- The join of $\mathcal{C}$ and $\mathcal{D} \in \mathcal{P}(N)$, denoted as $\mathcal{C} \vee \mathcal{D}$, is the finest cluster decomposition that is coarser than both $\mathcal{C}$ and $\mathcal{D}$.

We use the following partitions to decompose configuration space $M$ : given a subset $\emptyset \neq C \subseteq N$, we define the corresponding collision set as

$$
\Delta_{C}^{E}:=\left\{q \in M \mid q_{i}=q_{j} \text { if } i, j \in C\right\},
$$

and for a cluster decomposition $\mathcal{C}$ we define the $\mathcal{C}$-collision subspace

$$
\Delta_{\mathcal{C}}^{E}:=\left\{q \in M \mid q_{i}=q_{j} \text { if }[i]_{\mathcal{C}}=[j]_{\mathcal{C}}\right\}=\bigcap_{C \in \mathcal{C}} \Delta_{C}^{E} .
$$


By $\Pi_{C}^{E}$ we denote the $\mathcal{M}$-orthogonal projection onto the subspace $\Delta_{C}^{E}$, and we denote the complementary projection $\mathbb{1}_{\mathcal{M}}-\Pi_{C}^{E}$ by $\Pi_{C}^{I}$. Accordingly, we denote the (external) projection onto $\Delta_{\mathcal{C}}^{E}$ by $\Pi_{\mathcal{C}}^{E}:=\prod_{C \in \mathcal{C}} \Pi_{C}^{E}$, and the (internal) projection by $\Pi_{\mathcal{C}}^{I}=\mathbb{1}_{\mathcal{M}}-\Pi_{\mathcal{C}}^{E}=\sum_{C \in \mathcal{C}} \Pi_{C}^{I}$. The image of $\Pi_{C}^{I}$ is given by

$$
\Delta_{C}^{I}:=\left\{q \in M \mid \sum_{i \in C} m_{i} q_{i}=0, \forall i \in N \backslash C: q_{i}=0\right\},
$$

the image of $\Pi_{\mathcal{C}}^{I}$ is $\Delta_{\mathcal{C}}^{I}:=\left\{q \in M \mid \forall C \in \mathcal{C}: \sum_{i \in C} m_{i} q_{i}=0\right\}=\bigoplus_{C \in \mathcal{C}} \Delta_{C}^{I}$.

In particular, $\Delta_{\mathcal{C}_{\wedge}}^{E}=M$, see Def. 2.2. We get a $\mathcal{M}$-orthogonal decomposition

$$
M=\Delta_{\mathcal{C}}^{E} \oplus \bigoplus_{C \in \mathcal{C}} \Delta_{C}^{I}
$$

For a nonempty subset $C \subseteq N$ we define the cluster mass, cluster barycenter and cluster momentum of $C$ by

$$
m_{C}:=\sum_{j \in C} m_{j} \quad, \quad q_{C}:=\frac{1}{m_{C}} \sum_{j \in C} m_{j} q_{j} \quad \text { and } \quad p_{C}:=\sum_{i \in C} p_{i} .
$$

In particular $m_{N}$ equals the total mass of the particle system. Then for the partitions $\mathcal{C} \in \mathcal{P}(N)$ the $i$-th component of the cluster projection $q_{\mathcal{C}}^{E}:=\Pi_{\mathcal{C}}^{E}(q)$ is given by the barycenter, respectively its distance from the barycenter

$$
\left(q_{\mathcal{C}}^{E}\right)_{i}=q_{[i]_{\mathcal{C}}} \quad, \quad\left(q_{\mathcal{C}}^{I}\right)_{i}=q_{i}-q_{[i]_{\mathcal{C}}} \text { for } q_{\mathcal{C}}^{I}:=\Pi_{\mathcal{C}}^{I}(q) \quad(i \in N) .
$$

Join of partitions corresponds to intersection of collision subspaces:

$$
\Delta_{\mathcal{C}}^{E} \cap \Delta_{\mathcal{D}}^{E}=\Delta_{\mathcal{C} \vee \mathcal{D}}^{E} \quad(\mathcal{C}, \mathcal{D} \in \mathcal{P}(N)) .
$$

So for $\mathcal{C} \in \mathcal{P}(N)$, the mutually disjoint sets

$$
\Xi_{\mathcal{C}}^{(0)}:=\Delta_{\mathcal{C}}^{E} \backslash \bigcup_{\mathcal{D} \varsubsetneqq \mathcal{C}} \Delta_{\mathcal{D}}^{E}=\left\{q \in M \mid q_{i}=q_{j} \text { if and only if } i \sim_{\mathcal{C}} j\right\}
$$

form a set partition of $M$, with $\Xi_{\mathcal{C}_{\wedge}}^{(0)}=\widehat{M}$.

Denoting by $M^{*}$ the dual space of the vector space $M$, there are natural identifications $T M \cong M \times M, T^{*} M \cong M^{*} \times M$ of the tangent space resp. phase space of $M$. This gives rise to the inner products

$$
\langle\cdot, \cdot\rangle_{T M}: T M \times T M \rightarrow \mathbb{R} \quad, \quad\left\langle(q, v),\left(q^{\prime}, v^{\prime}\right)\right\rangle_{T M}:=\left\langle q, q^{\prime}\right\rangle_{\mathcal{M}}+\left\langle v, v^{\prime}\right\rangle_{\mathcal{M}}
$$

and

$\langle\cdot, \cdot\rangle_{T^{*} M}: T^{*} M \times T^{*} M \rightarrow \mathbb{R} \quad, \quad\left\langle(p, q),\left(q^{\prime}, p^{\prime}\right)\right\rangle_{T^{*} M}:=\left\langle q, q^{\prime}\right\rangle_{\mathcal{M}}+\left\langle p, p^{\prime}\right\rangle_{\mathcal{M}^{-1}}$ (with $\left\langle p, p^{\prime}\right\rangle_{\mathcal{M}^{-1}}=\sum_{i=1}^{n}\left\langle p_{i}, p_{i}^{\prime}\right\rangle / m_{i}$ for the momentum vector $p=\left(p_{1}, \ldots, p_{n}\right)$ ). 
The tangent space $T U$ of any subspace $U \subseteq M$ is naturally a subspace of $T M$. Using the inner product, we also consider $T^{*} U$ as a subspace of $T^{*} M$.

We thus obtain $T^{*} M$-orthogonal decompositions

$$
T^{*} M=T^{*} \Delta_{\mathcal{C}}^{E} \oplus \bigoplus_{C \in \mathcal{C}} T^{*}\left(\Delta_{C}^{I}\right) \quad(\mathcal{C} \in \mathcal{P}(N))
$$

of phase space. With $\widehat{\Pi}_{\mathcal{C}}^{I}:=\mathbb{1}_{T^{*} M}-\widehat{\Pi}_{\mathcal{C}}^{E}=\sum_{C \in \mathcal{C}} \widehat{\Pi}_{C}^{I}$ the $T^{*} M$-orthogonal projections $\widehat{\Pi}_{\mathcal{C}}^{E}, \widehat{\Pi}_{\mathcal{C}}^{I}: T^{*} M \rightarrow T^{*} M$ onto these subspaces are given by the cluster coordinates

$$
\left(p^{E}, q^{E}\right):=\widehat{\Pi}_{\mathcal{C}}^{E}(p, q) \quad \text { with } \quad\left(p_{i}, q_{i}\right)=\left(\frac{m_{i}}{m_{[i]}} p_{[i]}, q_{[i]}\right) \quad(i \in N),
$$

and relative coordinates

$$
\left(p^{I}, q^{I}\right):=\widehat{\Pi}_{\mathcal{C}}^{I}(p, q) \quad \text { with } \quad\left(p_{i}^{I}, q_{i}^{I}\right)=\left(p_{i}-p_{i}^{E}, q_{i}-q_{i}^{E}\right) \quad(i \in N) .
$$

Compared to (2.9) we omitted the subindex $\mathcal{C}$ in (2.11).

For nontrivial partitions neither the cluster coordinates nor the relative coordinates are coordinates in the strict sense. Later, however, we need such coordinates on the above-mentioned symplectic subspaces of phase space.

2.3 Lemma The vector space automorphisms

$$
\left(\widehat{\Pi}_{\mathcal{C}}^{E}, \widehat{\Pi}_{\mathcal{C}}^{I}\right): T^{*} M \longrightarrow T^{*} \Delta_{\mathcal{C}}^{E} \oplus \bigoplus_{C \in \mathcal{C}} T^{*}\left(\Delta_{C}^{I}\right) \quad(\mathcal{C} \in \mathcal{P}(N))
$$

are symplectic w.r.t. the natural symplectic forms on these cotangent bundles.

Proof. This follows from $T^{*}\left(\Delta_{\mathcal{C}}^{E} \oplus \bigoplus_{C \in \mathcal{C}} \Delta_{C}^{I}\right)=T^{*} \Delta_{\mathcal{C}}^{E} \oplus \bigoplus_{C \in \mathcal{C}} T^{*}\left(\Delta_{C}^{I}\right)$.

Total angular momentum

$$
L: T^{*} M \rightarrow \mathbb{R}^{d} \wedge \mathbb{R}^{d} \quad, \quad L(p, q)=\sum_{i=1}^{n} q_{i} \wedge p_{i}
$$

and total kinetic energy

$$
K: T^{*} M \rightarrow \mathbb{R} \quad, \quad K(p, q) \equiv K(p)=\frac{1}{2}\langle p, p\rangle_{\mathcal{M}^{*}}=\sum_{i=1}^{n} \frac{\left\langle p_{i}, p_{i}\right\rangle}{2 m_{i}}
$$

(using a sloppy notation) both split for $\mathcal{C} \in \mathcal{P}(N)$ into sums of barycentric

$$
\begin{aligned}
L_{\mathcal{C}}^{E}:=L \circ \widehat{\Pi}_{\mathcal{C}}^{E} \quad, \quad L_{\mathcal{C}}^{E}(p, q)=\sum_{C \in \mathcal{C}} q_{C} \wedge p_{C}, \\
K_{\mathcal{C}}^{E}:=K \circ \widehat{\Pi}_{\mathcal{C}}^{E} \quad, \quad K_{\mathcal{C}}^{E}(p, q)=\sum_{C \in \mathcal{C}} \frac{\left\langle p_{C}, p_{C}\right\rangle}{2 m_{C}}
\end{aligned}
$$

and relative terms for the clusters $C \in \mathcal{C}$

$$
\begin{aligned}
L_{C}^{I} & :=L \circ \widehat{\Pi}_{C}^{I} \quad, \quad L_{C}^{I}(p, q)=\sum_{i \in C} q_{i}^{I} \wedge p_{i}^{I} \\
K_{C}^{I} & :=K \circ \widehat{\Pi}_{C}^{I} \quad, \quad K_{C}^{I}(p, q)=\sum_{i \in C} \frac{\left\langle p_{i}^{I}, p_{i}^{I}\right\rangle}{2 m_{i}} .
\end{aligned}
$$


That is, $L=L_{\mathcal{C}}^{E}+\sum_{C \in \mathcal{C}} L_{C}^{I} \quad$ and $\quad K=K_{\mathcal{C}}^{E}+K_{\mathcal{C}}^{I} \quad$ with $\quad K_{\mathcal{C}}^{I}:=\sum_{C \in \mathcal{C}} K_{C}^{I}$.

The decomposition of the potential $V$ as a sum of external and internal terms is not given by composition with the corresponding projections.

Instead, we set $V_{C}:=\sum_{i<j \in C} V_{i, j}$ for $C \subseteq N$ and

$$
V_{\mathcal{C}}^{I}:=\sum_{C \in \mathcal{C}} V_{C} \quad, \quad V_{\mathcal{C}}^{E}:=V-V_{\mathcal{C}}^{I} \quad(\mathcal{C} \in \mathcal{P}(N)) .
$$

Finally, the Hamiltonian function $H$ decomposes into the terms

$$
H_{\mathcal{C}}^{I}:=\sum_{C \in \mathcal{C}} H_{C}^{I} \quad \text { with } \quad H_{C}^{I}:=K_{C}^{I}+V_{C}^{I} \quad(\mathcal{C} \in \mathcal{P}(N)),
$$

and similarly for $H_{C}^{E}$ and $H_{\mathcal{C}}^{E}$.

For simplifying notation, we sometimes omit the super-index $E$ (but not $I$ ).

\subsection{The wandering set}

We base our article on the observation that NAV is wandering, see Theorem 2.8. We shortly discuss the general method from [FK1] for $C^{1}$, volume-preserving dynamical systems $(P, \Omega, X)$, with $X: P \rightarrow T P$ having Lie derivative $\mathcal{L}_{X} \Omega=0$.

2.4 Definition The wandering set Wand $\subseteq P$ of the flow $\Phi$ consists of those $x \in P$ which have a neighborhood $U_{x}$ so that for a suitable time $t_{x} \in\left(0, T^{+}(x)\right)$

$$
U_{x} \cap \Phi\left(\left(\left(t_{x}, T^{+}(x)\right) \times U_{x}\right) \cap D\right)=\emptyset .
$$

As $\mathcal{L}_{X} \Omega=0, \Phi$ preserves the volume form $\Omega$. Consider now for the differential form of degree $\operatorname{dim}(P)-1$

$$
\mathcal{V}:=\boldsymbol{i}_{X} \Omega
$$

a sequence of hypersurfaces $\iota_{m}: \mathcal{H}_{m} \subseteq P(m \in \mathbb{N})$ which fulfill the Assumptions

(A1) to be transversal to $X$, so that $\mathcal{V}_{m}:=\iota_{m}^{*}(\mathcal{V})$ are volume forms on $\mathcal{H}_{m}$,

(A2) to have finite volumes $\left(\int_{\mathcal{H}_{m}} \mathcal{V}_{m}<\infty\right)$, and $\lim _{m \rightarrow \infty} \int_{\mathcal{H}_{m}} \mathcal{V}_{m}=0$.

$$
\text { Trans : }=\left\{x \in P \mid \exists m_{0} \in \mathbb{N} \forall m \geq m_{0}: \mathcal{O}^{+}(x) \cap \overline{\mathcal{H}}_{m} \neq \emptyset\right\}
$$

is the set of transition points, whose forward orbits eventually hit all of these surfaces. Then

\subsection{Theorem ([FK1], Theorem A) $\Omega($ Wand $\cap$ Trans $)=0$.}

So our task is to show that this method of Poincaré surfaces is applicable, i.e. setting $(P, \Omega, X):=\left(\Sigma_{E}, \sigma_{E},\left.X_{H}\right|_{\Sigma_{E}}\right)$ for energy $E \in \mathbb{R}$, (omitting indices $E$ )

(B1) to show that $\mathrm{NAV} \subseteq$ Wand, and

(B2) to define a sequence of hypersurfaces $\mathcal{H}_{m} \subseteq \Sigma_{E}$, fulfilling Assumptions $(\mathrm{A} 1)$ and $(\mathrm{A} 2)$, for which $\mathrm{NAV} \subseteq$ Trans. 


\subsection{Proof of assertion (B1)}

We consider the time evolution for the moment of inertia

$$
J: P \rightarrow \mathbb{R} \quad, \quad J(q):=\frac{1}{2} \sum_{i=1}^{n} m_{i}\left\|q_{i}\right\|^{2}=\frac{1}{2}\langle q, q\rangle_{\mathcal{M}}
$$

and use the following generalization by Fleischer [FI, Theorem 2.4.4] of von Zeipel's theorem [Ze, Sp]. As it stands, it is a result about individual orbits.

\subsection{Theorem (von Zeipel)}

For $n \in \mathbb{N}$ particles in d dimensions, a long ranged potential $V$ (see (1.2)) and

$$
\tilde{j}_{x}:\left(0, T^{+}(x)\right) \rightarrow[0, \infty) \quad, \quad \tilde{j}_{x}(t):=J(q(t, x) / t) \quad(x \in P)
$$

the limit $j^{+}(x):=\lim _{t \nearrow^{+}{ }^{+}(x)} \tilde{j}_{x}(t)$ exists in $[0, \infty] . j^{+}(x)$ is finite if and only if the asymptotic velocity $\bar{v}^{+}(x) \in \mathbb{R}^{d n}$ from (1.3) exists.

Indeed, in general the phase space function $j^{+}: P \rightarrow[0, \infty]$ is discontinuous.

\subsection{Example (Discontinuity of the phase space function $j^{+}$)}

1. For the Hamiltonian $H(p, q):=\frac{1}{2} p^{2}+V(q)$ with potential $V \in C_{c}^{\infty}(\mathbb{R}, \mathbb{R})$ of compact support and a unique absolute maximum $V(0)=1=: E, j^{+}$is not upper semicontinuous at $x_{0}:=(0,0) \in \Sigma_{E}$. Then $j^{+}\left(x_{0}\right)=0$, whereas for all initial conditions $x_{0}^{\prime}=\left(p_{0}^{\prime}, q_{0}^{\prime}\right) \in \Sigma_{E}$ with $p_{0}^{\prime} q_{0}^{\prime}>0$ one has $j^{+}\left(x_{0}^{\prime}\right)=\frac{1}{2}$.

2. For the $d \geq 2$-dimensional Kepler Hamiltonian $H(p, q):=\frac{1}{2}\|p\|^{2}-1 /\|q-c\|$ with $c \in \mathbb{R}^{d} \backslash\{0\}, j^{+}$is not lower semicontinuous at initial conditions $x_{0}=$ $\left(0, q_{0}\right)$ with $\left\|q_{0}-c\right\|=1$, that is, $E:=H\left(x_{0}\right)=-1$ and $x_{0} \in$ Coll. Then $j^{+}\left(x_{0}\right)=\frac{1}{2}\left\|c / T^{+}\left(x_{0}\right)\right\|^{2}$, but for all $x_{0}^{\prime} \in \Sigma_{E} \backslash$ Coll one has $j^{+}\left(x_{0}^{\prime}\right)=0$.

However, we have the following result, proven in Section 5.1, which is of independent interest and proves assertion (B1). It uses the time dependent cluster function $\mathcal{A}:(0, T(x)) \rightarrow \mathcal{P}(N)$, defined in Section 5.1, see (5.4).

\subsection{Theorem (Initial conditions without asymptotic velocities)}

For $n \in \mathbb{N}$ particles in $d \in \mathbb{N}$ dimensions and a long-ranged potential $V$,

1. For initial conditions $x \in \mathrm{NAV}$, the cluster-external speed has the limit $\lim _{t \nearrow T(x)}\left\|\frac{d}{d t} \tilde{q}_{\mathcal{A}(t)}^{E}(t)\right\|_{\mathcal{M}}=\infty$, so that $\lim _{t \nearrow T(x)} V(q(t, x))=-\infty$.

2. $j^{+}: P \rightarrow[0, \infty]$ is continuous ${ }^{1}$ at the points $x \in \mathrm{NAV}$.

3. Thus $\mathrm{NAV} \subseteq$ Wand, and $\mathrm{NAV} \subseteq P$ is a Borel set.

\footnotetext{
${ }^{1}$ regarding, as usual, $[0, \infty]$ as the one-point compactification of $[0, \infty)$
} 


\subsection{Proof of assertion (B2)}

Assertion (B2) on page 8 states that there is a sequence of hypersurfaces $\mathcal{H}_{m} \subseteq$ $\Sigma_{E}$, fulfilling Assumptions (A1) and (A2), for which $\mathrm{NAV}_{E} \subseteq$ Trans $_{E}$. We will perform two changes of that scheme, using the constants of motion $L$ and $p_{N}$. First consider total momentum and the center of mass, in the notation (2.8):

$$
p_{N}=\sum_{i \in N} p_{i} \quad \text { and } \quad q_{N}=\sum_{i \in N} \frac{m_{i}}{m_{N}} q_{i} \quad \text {, with } \quad m_{N}=\sum_{i \in N} m_{i} .
$$

Subsets of the energy surface in the center of mass frame

$$
\Sigma_{E, 0}:=\left\{(p, q) \in \Sigma_{E} \mid p_{N}=0, q_{N}=0\right\}
$$

are denoted by using the same indices $E, 0$.

2.9 Lemma (Center of mass) If $\mathrm{NAV}_{E, 0} \subseteq \Sigma_{E, 0}$ are of Liouville measure zero for all $E \in \mathbb{R}$, then $\mathrm{NAV}_{E} \subseteq \Sigma_{E}$ are of Liouville measure zero for all $E \in \mathbb{R}$.

Proof: This follows by symplectic reduction w.r.t. the free, proper Hamiltonian $\mathbb{R}^{d}$-action on $P$, being the symplectic lift of the diagonal $\mathbb{R}^{d}$-action on $\widehat{M}$ (acting trivially on the momenta).

Accordingly, we will work in the center of mass frame.

From a rough propagation estimate it follows that for $j^{+}(x)=\infty$, the cluster function $t \mapsto \mathcal{A}(t)$, see (5.4), must change its value infinitely often. We now show that for $n=4$ there is a messenger cluster moving between two others infinitely often as $t \nearrow T(x)$.

1. There is a nontrivial cluster: By Theorem 2.8, for $x \in \mathrm{NAV}_{E, 0}$ we have $\lim _{t \nearrow T(x)} \tilde{V}(t)=-\infty$. Choose $t_{0}^{\prime} \in(0, T(x))$ so that (with $\Xi_{\mathcal{C}}^{(\delta)}$ from (5.3))

$$
\tilde{V}(t)<\min \left\{V(r) \mid r \in\left(t_{0}^{\prime}\right)^{1-\varepsilon / 2} \Xi_{\mathcal{C}_{\curlywedge}}^{(\delta)}\right\} \quad\left(t \in\left[t_{0}^{\prime}, T(x)\right)\right) .
$$

This is possible, since the minimum is increasing in the parameter $t_{0}^{\prime}$.

Then by Definition (5.4) of $\mathcal{A}$, at least one atom of $\mathcal{A}(t)$ must be nontrivial for all $t \in\left[t_{0}, T(x)\right)$, so that $|\mathcal{A}(t)| \leq 3$.

2. There is more than one cluster: On the other hand, since $j^{+}(x)=\infty$, $\lim _{t \nearrow T(x)}\|q(t, x)\|_{\mathcal{M}} t^{\varepsilon / 2-1}=\infty$, too. As in the c.o.m. frame, the diameter of $\Xi_{\mathcal{C}_{\vee}}^{(\delta)}$ is finite, we can choose $t_{0}^{\prime \prime} \in(0, T(x))$ so that $t_{0}^{\varepsilon / 2-1} \notin \Xi_{\mathcal{C}_{\vee}}^{(\delta)}$ for $t \in\left[t_{0}^{\prime \prime}, T(x)\right)$. This implies that there are at least two clusters: $|\mathcal{A}(t)| \geq 2$.

For $t$ larger than $t_{0}:=\max \left(t_{0}^{\prime}, t_{0}^{\prime \prime}\right)$ the number $|\mathcal{A}(t)|$ of clusters is 2 or 3 . 
3. There are infinitely many changes between two and three clusters: If for some time $\tau \in\left[t_{0}, T(x)\right)$ one has $\mathcal{A}\left(\tau^{+}\right) \neq \mathcal{A}\left(\tau^{-}\right)$, then by construction of the Graf partition (5.3), $\left|\mathcal{A}\left(\tau^{+}\right)\right| \neq\left|\mathcal{A}\left(\tau^{-}\right)\right|$and $\mathcal{A}\left(\tau^{+}\right)$is comparable with $\mathcal{A}\left(\tau^{-}\right)$in the sense of Definition 2.2.

Breakup of a cluster followed by its recombination may happen.

However, there must occur infinitely many changes between non-comparable set partitions, in the following sense. There exists a strictly increasing sequence $\left(s_{k}\right)_{k \in \mathbb{N}}$ of $s_{k} \in\left[s_{0}, T(x)\right)$ with the properties $\mathcal{A}\left(s_{\ell}^{+}\right) \neq \mathcal{A}\left(s_{\ell}^{-}\right)$, $\left.\mathcal{A}\right|_{\left(s_{\ell}, s_{\ell+1}\right)}$ constant, that $\left|\mathcal{A}\left(s_{\ell}^{+}\right)\right|=3$ if $\ell$ is even and $\left|\mathcal{A}\left(s_{\ell}^{+}\right)\right|=2$ if $\ell$ is odd, and for every odd $\ell$ there is a minimal odd $\ell^{\prime}>\ell$ with $\mathcal{A}\left(s_{\ell^{\prime}}^{+}\right)$not comparable with $\mathcal{A}\left(s_{\ell}^{+}\right)$.

Otherwise $j^{+}(x)$ would be finite, since then the term $\frac{1}{2 t^{2}}\left\|\tilde{Q}_{\mathcal{A}}^{E}-\tilde{v}_{\mathcal{A}}^{E}\right\|_{\mathcal{M}}^{2}$ in

$$
\frac{d^{2}}{d t^{2}} \tilde{j}^{E}=-\frac{4}{t^{2}}\left\langle\tilde{Q}^{E}, \frac{d}{d t} \tilde{q}^{E}-\tilde{Q}^{E}\right\rangle_{\mathcal{M}}+\frac{1}{2 t^{2}}\left\|\tilde{Q}_{\mathcal{A}}^{E}-\tilde{v}_{\mathcal{A}}^{E}\right\|_{\mathcal{M}}^{2}-\frac{2}{t^{2}}\left\langle\tilde{q}^{E}, \nabla \tilde{V}^{E}\right\rangle
$$

would be twice integrable, the two other terms having this property unconditionally (compare with $\frac{d}{d t} \tilde{j}^{E}$ in (5.5)).

4. Symbolic description: From this sequence we extract a subsequence $\left(t_{k}\right)_{k \in \mathbb{N}}$, $t_{k}=s_{\ell(k)}$, maximal with respect to the properties that for $k$ even,

- $\mathcal{A}$ is constant in the interval $\left(t_{k}, t_{k+1}\right)$, and of size $\left|\mathcal{A}\left(t_{k}^{+}\right)\right|=3$,

- $\ell(k \pm 1)=\ell(k) \pm 1,\left|\mathcal{A}\left(t_{k}^{-}\right)\right|=\left|\mathcal{A}\left(t_{k+1}^{+}\right)\right|=2$ and $\mathcal{A}\left(t_{k}^{-}\right)$and $\mathcal{A}\left(t_{k+1}^{+}\right)$are not comparable.

Note that then for $k$ odd, $\mathcal{A}$ need not to be constant in the interval $\left(t_{k}, t_{k+1}\right)$, but by maximality $\mathcal{A}\left(t_{k}^{+}\right)=\mathcal{A}\left(t_{k+1}^{-}\right)$, consisting of two clusters.

For $k$ even we consider the three-tuple $\left(\mathcal{C}_{k-1}, \mathcal{C}_{k}, \mathcal{C}_{k+1}\right)$ with $\mathcal{C}_{\ell}:=\mathcal{A}\left(t_{\ell}^{+}\right)$. So $\left|\mathcal{C}_{k}\right|=3,\left|\mathcal{C}_{k \pm 1}\right|=2, \mathcal{C}_{k}$ is comparable with both $\mathcal{C}_{k \pm 1}$, but $\mathcal{C}_{k-1}$ is not comparable with $\mathcal{C}_{k+1}$. Thus we can uniquely enumerate the clusters of $\mathcal{C}_{k}$ in such a way that for $\mathcal{C}_{k}=\left\{C_{1}, C_{2}, C_{3}\right\}$ we have

$$
\mathcal{C}_{k-1}=\left\{C_{1} \cup C_{2}, C_{3}\right\} \quad \text { and } \quad \mathcal{C}_{k+1}=\left\{C_{1}, C_{2} \cup C_{3}\right\} .
$$

So $C_{2}$ is a cluster of particles whose center $\tilde{q}_{C_{2}}$ moves in the interval $\left(t_{k}, t_{k+1}\right)$ from a neighborhood of $\tilde{q}_{C_{1}}\left(t_{k}\right)$ to one of $\tilde{q}_{C_{3}}\left(t_{k+1}\right)$. It is called the messenger.

5. Combinatorics: For $n=4$ the set $\mathcal{Q}$ of three three-tuples $\left(C_{1}, C_{2}, C_{3}\right)$ of clusters with $\left\{C_{1}, C_{2}, C_{3}\right\} \in \mathcal{P}_{3}(N)$ (see (2.5)) is of size $|\mathcal{Q}|=\left(\begin{array}{l}4 \\ 2\end{array}\right) 3 !=36$. 
Propagation estimates show (see Section 4.3) that between their near-collisions, the three cluster centers move asymptotically as $t \nearrow T(x)$ on straight lines in $\mathbb{R}^{d}$.

Thus we first consider in the following section 3 a nondeterministic kinematical model of three particles moving on straight lines between their collisions. Then in Section 4 we show that asymptotically the dynamics is indeed captured by that model. Finally, in Section 5.2 we define Poincaré surfaces which fulfill Assumptions (A1) and (A2) (Lemma 5.2), with $\mathrm{NAV}_{E} \subseteq$ Trans $_{E}$.

\section{A nondeterministic kinematical model}

Asymptotically, as $t \nearrow T(x)$, between their near-collisions the three cluster centers $\tilde{q}_{C_{i}}$ move on straight lines. So to understand the kinematics, we set up a simple model, partly resembling the one of [FKM].

- Three points with positions $\tilde{q}_{1} \leq \tilde{q}_{2} \leq \tilde{q}_{3} \in C^{0}\left([0, T), \mathbb{R}^{d}\right)$ move with constant velocities $\tilde{v}_{i}=\frac{d}{d t} \tilde{q}_{i}$ in configuration space $\mathbb{R}^{d}$, until exactly two of them (say, $i$ and $j$ ) collide at collision times $t_{k} \in[0, T)$, that is, $\tilde{q}_{i}\left(t_{k}\right)=\tilde{q}_{j}\left(t_{k}\right)$.

- The number of collisions happening in the future is infinite. Collisions are enumerated by $k \in \mathbb{N}$, with $t_{1}:=0, t_{k+1}>t_{k}$ and $\lim _{k \rightarrow \infty} t_{k}=T$.

For $k$ even (odd), particle 2 collides with 3 (respectively 1 ) at time $t_{k}$.

- Their masses may depend on time:

$$
\widetilde{m}_{i}: \mathcal{T} \rightarrow\left[m_{\min }, m_{\text {max }}\right] \quad \text { with } \mathcal{T}:=[0, T) \backslash \cup_{k \in \mathbb{N}}\left\{t_{k}\right\} \text { and } m_{\text {min }}>0 .
$$

They are constant between collisions. The masses of the colliding particles may change during collision, but their sum is conserved:

$$
\widetilde{m}_{i}\left(t_{k}^{+}\right)+\widetilde{m}_{j}\left(t_{k}^{+}\right)=\widetilde{m}_{i}\left(t_{k}^{-}\right)+\widetilde{m}_{j}\left(t_{k}^{-}\right) .
$$

So the total mass $M:=\widetilde{m}_{1}+\widetilde{m}_{2}+\widetilde{m}_{3}$ is constant.

- Denoting by $\tilde{p}_{i}:=\widetilde{m}_{i} \tilde{v}_{i}$ their momenta, during collision of particles $i$ and $j$, their external momentum is constant:

$$
\tilde{p}_{i}\left(t_{k}^{+}\right)+\tilde{p}_{j}\left(t_{k}^{+}\right)=\tilde{p}_{i}\left(t_{k}^{-}\right)+\tilde{p}_{j}\left(t_{k}^{-}\right) .
$$

So by (3.1) at time $t_{k}$ their center of mass moves with constant velocity. But the internal momentum $\frac{\tilde{m}_{j} \tilde{p}_{i}-\tilde{m}_{i} \tilde{p}_{j}}{\tilde{m}_{i}+\tilde{m}_{j}}$ may change arbitrarily, without conservation of total energy

$$
\tilde{K}:=\sum_{i=1}^{3} \frac{\left\|\tilde{p}_{i}\right\|^{2}}{2 \tilde{m}_{i}}: \mathcal{T} \rightarrow \mathbb{R}
$$


- The initial conditions are chosen so that for $k=1$, that is, $t_{1}=0$ one has $\tilde{J}^{\prime}\left(t_{k}^{+}\right)>0$ for the moment of inertia

$$
\tilde{J}:=\frac{1}{2} \sum_{i=1}^{3} \tilde{m}_{i}\left\|\tilde{q}_{i}\right\|^{2}:[0, T) \rightarrow \mathbb{R} .
$$

For application of the model to non-collision singularities, we would have $T \in$ $(0,+\infty)$. For modeling orbits having no asymptotic velocity, we take $T:=+\infty$.

The model indeed catches some properties of the 4-body orbits whose asymptotic velocity does not exist. For example, particle 2 models the messenger cluster $C_{2}$ moving between $C_{1}$ and $C_{3}$. Of which of the four physical particles these three clusters consist, may change during near-collisions between three particles (so one would set $\left.\widetilde{m}_{i}(t):=m_{C_{i}(t)}\right)$. Also, the cluster $D(t)$ consisting of two particles serves as an infinite reservoir of kinetic cluster energy.

\subsection{Proposition (Nondeterministic kinematical model)}

1. The total angular momentum $\tilde{L}:=\sum_{i=1}^{3} \tilde{L}_{i}$ with $\tilde{L}_{i}:=\tilde{q}_{i} \wedge \tilde{p}_{i}$ is conserved. In the center of mass frame, $\tilde{L}$ is zero or of rank two. So the motion takes place on a line or a two-plane in configuration space $\mathbb{R}^{d}$.

Considered as functions $\tilde{L}_{i}: \mathcal{T} \rightarrow \mathbb{R}$, they are locally constant, and

$$
\left|\tilde{L}_{i}\right| \leq|\tilde{L}| \quad(i=1,2,3) .
$$

2. The moment of inertia $\tilde{J}$, see $(3.4)$, is in $C^{1}([0, T), \mathbb{R})$, with $\frac{d^{2}}{d t^{2}} \tilde{J}(t)=$ $2 \tilde{K}(t)>0$ for $t \in \mathcal{T}$. Thus

$$
\tilde{J}^{\prime}\left(t_{k+1}^{+}\right)=\tilde{J}^{\prime}\left(t_{k}^{+}\right)+2\left(t_{k+1}-t_{k}\right) \tilde{K}\left(t_{k}^{+}\right) .
$$

Moreover, for $\lambda:=\left(1+\frac{m_{\min }}{m_{\max }}\right)^{1 / 2}>1$ and some $J_{0}>0$

$$
\tilde{J}\left(t_{k}\right) \geq \lambda^{k} J_{0} \quad(k \in \mathbb{N}) .
$$

3. The speeds $\left\|\tilde{v}_{i}\left(t_{k}^{+}\right)\right\|$are non-zero for all $k \in \mathbb{N}$, and

$$
\left\langle\tilde{p}_{1}\left(t_{k}^{+}\right), \tilde{q}_{1}\left(t_{k}\right)\right\rangle>0,\left\langle\tilde{p}_{2}\left(t_{k}^{+}\right), \tilde{q}_{2}\left(t_{k}\right)\right\rangle<0 \text { and }\left\langle\tilde{p}_{3}\left(t_{k}^{+}\right), \tilde{q}_{3}\left(t_{k}\right)\right\rangle>0 \text {. }
$$

4. The total kinetic energy $\tilde{K}\left(t_{k}^{+}\right)$goes to infinity w.r.t. the collision index $k$ : For $\mu:=1+\left(\frac{m_{\min }}{m_{\max }}\right)^{2}>1$ and some $K_{0}>0$ depending on the initial conditions

$$
\tilde{K}\left(t_{k}^{+}\right) \geq \mu^{k} K_{0} \quad(k \in \mathbb{N}) .
$$


5. The directions $\hat{v}_{i}:=\tilde{v}_{i} /\left\|\tilde{v}_{i}\right\|$ align in the following sense: The limits

$$
w_{i}^{e}:=\lim _{k \rightarrow \infty} \hat{v}_{i}\left(t_{2 k}^{+}\right) \in S^{1} \quad \text { and } \quad w_{i}^{o}:=\lim _{k \rightarrow \infty} \hat{v}_{i}\left(t_{2 k+1}^{+}\right) \in S^{1} \quad(i=1,2,3)
$$

exist (index indicating even and odd), and $w_{1}^{e}=w_{1}^{o}=w_{2}^{e}=-w_{2}^{o}=w_{3}^{o}=w_{3}^{e}$. The speed of convergence is exponential in $k$.

\subsection{Remark (A tale of two scales)}

The exponential lower bounds (3.7) and (3.9) depend on collision index $k \in \mathbb{N}$ instead of time $t_{k}$. From (3.6) one infers that, depending on the sequence $k \mapsto t_{k+1}-t_{k}$ of time differences, the true behavior of these functions can be super-exponential in $k$, and the growth of spatial extension is not coupled to the increase in speed. Celestial bodies on a line show the same behavior, see [SX]. $\diamond$

\section{Proof:}

1. $\tilde{L}$ is constant, since between collisions its derivative vanishes, and during collision of particle $i$ and $j$ at time $t_{k}$ we have $\tilde{q}_{i}\left(t_{k}\right)=\tilde{q}_{j}\left(t_{k}\right)$ so that

$$
\begin{aligned}
\tilde{q}_{i}\left(t_{k}\right) & \wedge \tilde{p}_{i}\left(t_{k}^{-}\right)+\tilde{q}_{j}\left(t_{k}\right) \wedge \tilde{p}_{j}\left(t_{k}^{-}\right)=\tilde{q}_{i}\left(t_{k}\right) \wedge\left(\tilde{p}_{i}\left(t_{k}^{-}\right)+\tilde{p}_{j}\left(t_{k}^{-}\right)\right) \\
& =\tilde{q}_{i}\left(t_{k}\right) \wedge\left(\tilde{p}_{i}\left(t_{k}^{+}\right)+\tilde{p}_{j}\left(t_{k}^{+}\right)\right)=\tilde{q}_{i}\left(t_{k}\right) \wedge \tilde{p}_{i}\left(t_{k}^{+}\right)+\tilde{q}_{j}\left(t_{k}\right) \wedge \tilde{p}_{j}\left(t_{k}^{+}\right) .
\end{aligned}
$$

For $k$ odd, at times $t_{k}<t_{k+1}<t_{k+2}$ particle two collides first with particle one, then with three and again with one. In the inertial frame where $\tilde{q}_{1}(t)=0$ for $t \in\left(t_{k}, t_{k+2}\right)$, the momenta $\tilde{p}_{2}\left(t_{k}^{+}\right)$and $\tilde{p}_{3}\left(t_{k}^{+}\right)$span a line or plane. For having the collision at time $t_{k+2}, \tilde{p}_{2}\left(t_{k+1}^{+}\right)$must be linear dependent on $\tilde{p}_{2}\left(t_{k+1}^{-}\right)=\tilde{p}_{2}\left(t_{k}^{+}\right)$. So also $\tilde{p}_{3}\left(t_{k+1}^{+}\right)$must be in that line or plane, as is the center of mass. The case of $k$ even is similar. Thus $\operatorname{rank}(\tilde{L}) \leq 2$.

Next we derive (3.5). It is clear that the $\tilde{L}_{i}$ are locally constant, so that $\tilde{L}_{i}\left(t_{k+1}^{-}\right)=\tilde{L}_{i}\left(t_{k}^{+}\right)$. As real-valued functions on $T^{*} E \cong \mathbb{R}^{2} \times \mathbb{R}^{2}$, the $L_{i}$ have the presentations $L_{i}=\left\langle\mathbb{J} q_{i}, p_{i}\right\rangle$, with $\mathbb{J}:=\left(\begin{array}{cc}0 & -1 \\ 1 & 0\end{array}\right)$. We consider the case of $k \in \mathbb{N}$ odd (the case of even $k$ being similar).

(a) Solving for the momentum of the messenger particle two,

$$
\tilde{p}_{2}\left(t_{k}^{+}\right)=\widetilde{m}_{2}\left(t_{k}^{+}\right) \frac{\tilde{q}_{3}\left(t_{k}\right)-\tilde{q}_{2}\left(t_{k}\right)}{t_{k+1}-t_{k}}+\frac{\widetilde{m}_{2}\left(t_{k}^{+}\right)}{\widetilde{m}_{3}\left(t_{k}^{+}\right)} \tilde{p}_{3}\left(t_{k}^{+}\right) .
$$

We have $\tilde{q}_{1}\left(t_{k}\right)=\tilde{q}_{2}\left(t_{k}\right)$. Therefore in the center of mass frame $\tilde{q}_{3}\left(t_{k}\right)$ is a multiple of $\tilde{q}_{2}\left(t_{k}\right)$. So the first term in (3.11) does not contribute to $\tilde{L}_{2}\left(t_{k}^{+}\right)$, and we get

$$
\tilde{L}_{2}\left(t_{k}^{+}\right)=\frac{\widetilde{m}_{2}\left(t_{k}^{+}\right)}{\widetilde{m}_{3}\left(t_{k}^{+}\right)}\left\langle\mathbb{d} \tilde{q}_{2}\left(t_{k}^{+}\right), \tilde{p}_{3}\left(t_{k}^{+}\right)\right\rangle=-\frac{\widetilde{m}_{2}\left(t_{k}^{+}\right)}{\widetilde{m}_{1}\left(t_{k}^{+}\right)+\widetilde{m}_{2}\left(t_{k}^{+}\right)} \tilde{L}_{3}\left(t_{k}^{+}\right) .
$$


(b) Concerning particle one, we use that $\tilde{q}_{1}\left(t_{k}\right)=\tilde{q}_{2}\left(t_{k}\right)$ and $\tilde{p}_{1}=-\tilde{p}_{2}-\tilde{p}_{3}$ :

$$
\begin{aligned}
\tilde{L}_{1}\left(t_{k}^{+}\right) & =\left\langle\mathbb{d} \tilde{q}_{1}\left(t_{k}^{+}\right), \tilde{p}_{1}\left(t_{k}^{+}\right)\right\rangle=-\tilde{L}_{2}\left(t_{k}^{+}\right)-\left\langle d \tilde{\Phi} \tilde{q}_{1}\left(t_{k}^{+}\right), \tilde{p}_{3}\left(t_{k}^{+}\right)\right\rangle \\
& =\frac{\widetilde{m}_{2}\left(t_{k}^{+}\right)+\widetilde{m}_{3}\left(t_{k}^{+}\right)}{\widetilde{m}_{1}\left(t_{k}^{+}\right)+\widetilde{m}_{2}\left(t_{k}^{+}\right)} \tilde{L}_{3}\left(t_{k}^{+}\right) .
\end{aligned}
$$

(c) Thus $\tilde{L}=\tilde{L}_{1}\left(t_{k}^{+}\right)+\tilde{L}_{2}\left(t_{k}^{+}\right)+\tilde{L}_{3}\left(t_{k}^{+}\right)=\frac{M}{M-\widetilde{m}_{3}\left(t_{k}^{+}\right)} \tilde{L}_{3}\left(t_{k}^{+}\right)$, or

$$
\tilde{L}_{1}\left(t_{k}^{+}\right)=\frac{M-\widetilde{m}_{1}\left(t_{k}^{+}\right)}{M} \tilde{L}, \tilde{L}_{2}\left(t_{k}^{+}\right)=-\frac{\widetilde{m}_{2}\left(t_{k}^{+}\right)}{M} \tilde{L} \text { and } \tilde{L}_{3}\left(t_{k}^{+}\right)=\frac{M-\widetilde{m}_{3}\left(t_{k}^{+}\right)}{M} \tilde{L} .
$$

In particular, if the angular momentum $\tilde{L}(0)=0$ then for all $k$ the affine lines

$$
\ell_{i}(k):=\tilde{q}_{i}\left(t_{k}^{+}\right)+\operatorname{span}\left(\tilde{p}_{i}\left(t_{k}^{+}\right)\right) \quad(i=1,2,3),
$$

on which the particles move in the time interval $\left[t_{k}, t_{k+1}\right]$, coincide.

2. For non-collision times $t \in \mathcal{T}$

$$
\frac{d}{d t} \tilde{J}(t)=\sum_{i=1}^{3}\left\langle\tilde{q}_{i}, \tilde{p}_{i}\right\rangle(t) \quad \text { and } \quad \frac{d^{2}}{d t^{2}} \tilde{J}(t)=2 \tilde{K}(t) .
$$

By (3.2), for collision of particles $i$ and $j$ at time $t_{k}$

$$
\left\langle\tilde{p}_{i}\left(t_{k}^{+}\right), \tilde{q}_{i}\left(t_{k}\right)\right\rangle+\left\langle\tilde{p}_{j}\left(t_{k}^{+}\right), \tilde{q}_{j}\left(t_{k}\right)\right\rangle=\left\langle\tilde{p}_{i}\left(t_{k}^{-}\right), \tilde{q}_{i}\left(t_{k}\right)\right\rangle+\left\langle\tilde{p}_{j}\left(t_{k}^{-}\right), \tilde{q}_{j}\left(t_{k}\right)\right\rangle .
$$

Thus we can uniquely extend its derivative so that $\tilde{J} \in C^{1}([0, T), \mathbb{R})$, and its second derivative exists on $\mathcal{T}$ and is locally constant.

Equation (3.6) follows with (3.15) for all $k \in \mathbb{N}$, since by (3.14)

$$
\sum_{i=1}^{3}\left\langle\tilde{p}_{i}\left(t_{k+1}^{-}\right), \tilde{q}_{i}\left(t_{k+1}\right)\right\rangle=\sum_{i=1}^{3}\left\langle\tilde{p}_{i}\left(t_{k}^{+}\right), \tilde{q}_{i}\left(t_{k}\right)\right\rangle+2\left(t_{k+1}-t_{k}\right) \tilde{K}\left(t_{k}^{+}\right),
$$

and $\tilde{K}\left(t_{k}^{+}\right)>0$, since otherwise there would not be a further collision.

To prove (3.7), we first analyze the special case of motion on a line. Then both (3.2), the motion between collisions

$$
\tilde{q}_{i}\left(t_{k+1}\right)=\tilde{q}_{i}\left(t_{k}\right)+\frac{\Delta t_{k}}{\widetilde{m}_{i}\left(t_{k}^{+}\right)} \tilde{p}_{i}\left(t_{k}^{+}\right) \quad(k \in \mathbb{N}, i=1,2,3)
$$

and the collision conditions

$$
\tilde{p}_{2}\left(t_{k}^{+}\right)=\frac{\tilde{m}_{2}\left(t_{k}^{+}\right)}{\Delta t_{k}} \times \begin{cases}\left(\tilde{q}_{3}\left(t_{k+1}\right)-\tilde{q}_{1}\left(t_{k}\right)\right) & , k \text { odd } \\ \left(\tilde{q}_{1}\left(t_{k+1}\right)-\tilde{q}_{3}\left(t_{k}\right)\right) & , k \text { even }\end{cases}
$$

at times $t_{k}$ are linear in the $\tilde{q}_{i}\left(t_{k}\right)$ and $\tilde{p}_{i}\left(t_{k}^{+}\right)$, the coefficients being rational functions in the time differences $\Delta t_{k}:=t_{k+1}-t_{k}>0$ and the masses $\widetilde{m}_{i}\left(t_{k}^{+}\right)$. 
For $\Delta \tilde{q}:=\tilde{q}_{3}-\tilde{q}_{1}$ we obtain for two subsequent collisions a somewhat large set of linear equations in the positions and momenta at times $t_{k}$ and $t_{k+2}$. As we know the sign of its coefficients, assuming (3.8) (whose proof will be not use estimate (3.7)), without writing the equations explicitly, we have

$$
\Delta \tilde{q}\left(t_{k+2}\right) \geq\left\{\begin{array}{ll}
\left(1+\frac{\widetilde{m}_{2}\left(t_{k+1}^{+}\right)}{\widetilde{m}_{1}\left(t_{k+1}^{+}\right)}\right) \Delta \tilde{q}\left(t_{k}\right), k \text { odd } \\
\left(1+\frac{\widetilde{m}_{2}\left(t_{k+1}^{+}\right)}{\widetilde{m}_{3}\left(t_{k+1}^{+}\right)}\right) \Delta \tilde{q}\left(t_{k}\right), k \text { even }
\end{array} .\right.
$$

Since $\Delta \tilde{q}\left(t_{1}\right)>0$, this proves estimate (3.7), with $\lambda=\left(1+\frac{m_{\min }}{m_{\max }}\right)^{1 / 2}$.

For the generic case of non-vanishing angular momentum $\tilde{L}$, we already know that the motion in the plane is asymptotic to a one on a line. So by a continuity argument we get the result also in the general case.

3. That the speeds $\left\|\tilde{v}_{i}\left(t_{k}^{+}\right)\right\|$are non-zero follows from the inequalities (3.8). We show that these are valid for all $k \in \mathbb{N}$, assuming w.l.o.g. that $k$ is odd.

(a) As $\tilde{q}_{1}\left(t_{k}\right)=\tilde{q}_{2}\left(t_{k}\right)$, in the center of mass system

$$
0<\tilde{J}^{\prime}\left(t_{k}\right)=\sum_{i=1}^{3}\left\langle\tilde{p}_{i}\left(t_{k}^{+}\right), \tilde{q}_{i}\left(t_{k}\right)\right\rangle=\left\langle\tilde{p}_{3}\left(t_{k}^{+}\right), \tilde{q}_{3}\left(t_{k}\right)\right\rangle \frac{M}{\tilde{m}_{3}\left(t_{k}^{+}\right)},
$$

showing the third inequality in (3.8). Additionally we get for $i=1,2$

$$
\tilde{q}_{i}\left(t_{k}\right)=-\frac{\widetilde{m}_{3}\left(t_{k}^{+}\right)}{M-\widetilde{m}_{3}\left(t_{k}^{+}\right)} \tilde{q}_{3}\left(t_{k}^{+}\right) \quad \text { and thus } \quad\left\langle\tilde{p}_{3}\left(t_{k}^{+}\right), \tilde{q}_{i}\left(t_{k}\right)\right\rangle=-\frac{M \tilde{J}^{\prime}\left(t_{k}\right)}{M-\widetilde{m}_{3}\left(t_{k}^{+}\right)} .
$$

(b) We now take the next collision at time $t_{k+1}$ and between particles 2 and 3 into account. By (3.11) and both identities in (3.17)

$$
\left\langle\tilde{p}_{2}\left(t_{k}^{+}\right), \tilde{q}_{2}\left(t_{k}\right)\right\rangle=-\frac{\widetilde{m}_{2} M}{M-\widetilde{m}_{3}}\left(\frac{\widetilde{m}_{3}}{M-\widetilde{m}_{3}} \frac{\left\|\tilde{q}_{3}\right\|^{2}}{t_{k+1}-t_{k}}+\frac{\tilde{J}^{\prime}}{\widetilde{m}_{3}}\right)\left(t_{k}^{+}\right)<0 .
$$

(c) To prove the first inequality in (3.8), we note that by the above

$$
\left\langle\tilde{p}_{1}\left(t_{k}^{+}\right), \tilde{q}_{1}\left(t_{k}\right)\right\rangle=-\left\langle\tilde{p}_{2}\left(t_{k}^{+}\right), \tilde{q}_{2}\left(t_{k}\right)\right\rangle-\left\langle\tilde{p}_{3}\left(t_{k}^{+}\right), \tilde{q}_{2}\left(t_{k}\right)\right\rangle>0 .
$$

4. With $\hat{q}_{i}:=\tilde{q}_{i} /\left\|\tilde{q}_{i}\right\|$, at collision times $t_{k}$ we estimate from below the terms

$$
\tilde{K}_{\|}: \mathcal{T} \rightarrow \mathbb{R} \quad, \quad \tilde{K}_{\|}:=\frac{\left\langle\tilde{p}_{1}, \hat{q}_{1}\right\rangle^{2}+\left\langle\tilde{p}_{3}, \hat{q}_{3}\right\rangle^{2}}{2 M}
$$

in the kinetic energies, corresponding to the momentum components that are then parallel to the line through the positions of all three particles. 
We know from (3.8) that $\tilde{K}_{\|}\left(t_{1}^{+}\right)>0$. Also $\tilde{K}_{\|}<\tilde{K}$, since, unlike in (3.3), the mass $M$ appears in (3.19), and $\widetilde{m}_{i}<M$. We prove that

$$
\tilde{K}_{\|}\left(t_{k+1}^{+}\right) \geq \mu \tilde{K}_{\|}\left(t_{k}^{+}\right) \text {with } \quad \mu=1+\left(\frac{m_{\min }}{m_{\max }}\right)^{2}>1
$$

assuming w.I.o.g. that $k$ is odd. Then (3.9) follows with $K_{0}:=\tilde{K}_{\|}\left(t_{1}^{+}\right) / \mu$.

(a) The term

$$
C_{1}(k):=\frac{\left\langle\tilde{p}_{1}\left(t_{k+1}^{+}\right), \hat{q}_{1}\left(t_{k+1}\right)\right\rangle^{2}}{2 M}-\frac{\left\langle\tilde{p}_{1}\left(t_{k}^{+}\right), \hat{q}_{1}\left(t_{k}\right)\right\rangle^{2}}{2 M}
$$

in $\tilde{K}_{\|}\left(t_{k+1}^{+}\right)-\tilde{K}_{\|}\left(t_{k}^{+}\right)$is positive, since $\tilde{p}_{1}\left(t_{k+1}^{+}\right)=\tilde{p}_{1}\left(t_{k}^{+}\right)$and

$$
\tilde{q}_{1}\left(t_{k+1}\right)=\tilde{q}_{1}\left(t_{k}\right)+\frac{\tilde{p}_{1}\left(t_{k}^{+}\right)}{\tilde{m}_{1}\left(t_{k}^{+}\right)}\left(t_{k+1}-t_{k}\right) \text { with } t_{k+1}-t_{k}>0,
$$

so that $\hat{q}_{1}\left(t_{k+1}\right)$ is more aligned to $\tilde{p}_{1}\left(t_{k}^{+}\right)$than $\hat{q}_{1}\left(t_{k}\right)$.

(b) The second term in $\tilde{K}_{\|}\left(t_{k+1}^{+}\right)-\tilde{K}_{\|}\left(t_{k}^{+}\right)$,

$$
C_{3}(k):=\frac{\left\langle\tilde{p}_{3}\left(t_{k+1}^{+}\right), \hat{q}_{3}\left(t_{k+1}\right)\right\rangle^{2}}{2 M}-\frac{\left\langle\tilde{p}_{3}\left(t_{k}^{+}\right), \hat{q}_{3}\left(t_{k}\right)\right\rangle^{2}}{2 M},
$$

is more complicated and will be estimated more precisely. We have

$$
\tilde{p}_{3}\left(t_{k+1}^{+}\right)=\tilde{p}_{3}\left(t_{k+1}^{-}\right)+\left(\tilde{p}_{2}\left(t_{k+1}^{-}\right)-\tilde{p}_{2}\left(t_{k+1}^{+}\right)\right)
$$

and $\tilde{p}_{i}\left(t_{k+1}^{-}\right)=\tilde{p}_{i}\left(t_{k}^{+}\right)$. Thus

$$
C_{3}(k)=\frac{\left(D_{3, I}^{a}(k)+D_{3, I I}(k)+D_{3, I I I}(k)\right)^{2}-\left(D_{3, I}^{b}(k)\right)^{2}}{2 M}
$$

with

$$
\begin{aligned}
& D_{3, I}^{a}(k):=\left\langle\tilde{p}_{3}\left(t_{k}^{+}\right), \hat{q}_{3}\left(t_{k+1}\right)\right\rangle, D_{3, I}^{b}(k):=\left\langle\tilde{p}_{3}\left(t_{k}^{+}\right), \hat{q}_{3}\left(t_{k}\right)\right\rangle \\
& D_{3, I I}(k):=\left\langle\tilde{p}_{2}\left(t_{k}^{+}\right), \hat{q}_{3}\left(t_{k+1}\right)\right\rangle \text { and } \\
& D_{3, I I I}(k):=-\left\langle\tilde{p}_{2}\left(t_{k+1}^{+}\right), \hat{q}_{3}\left(t_{k+1}\right)\right\rangle .
\end{aligned}
$$

I. By (3.16) and the same argument as in (a), one has

$$
D_{3, I}^{a}(k) \geq D_{3, I}^{b}(k)>0 \text {. }
$$

II. By solving the identity $\frac{\tilde{p}_{2}\left(t_{k}^{+}\right)}{\widetilde{m}_{2}\left(t_{k}^{+}\right)}\left(t_{k+1}-t_{k}\right)=\tilde{q}_{2}\left(t_{k+1}\right)-\tilde{q}_{2}\left(t_{k}\right)=$

$$
\begin{aligned}
\tilde{q}_{3}\left(t_{k+1}\right) & -\tilde{q}_{1}\left(t_{k}\right)=\tilde{q}_{3}\left(t_{k}\right)-\tilde{q}_{1}\left(t_{k}\right)+\frac{\tilde{p}_{3}\left(t_{k}^{+}\right)}{\widetilde{m}_{3}\left(t_{k}^{+}\right)}\left(t_{k+1}-t_{k}\right) \text { for } \tilde{p}_{2}\left(t_{k}^{+}\right), \\
D_{3, I I}(k) & =\widetilde{m}_{2}\left(t_{k}^{+}\right)\left\langle\frac{\tilde{q}_{3}\left(t_{k}\right)-\tilde{q}_{1}\left(t_{k}\right)}{t_{k+1}-t_{k}}+\frac{\tilde{p}_{3}\left(t_{k}^{+}\right)}{\widetilde{m}_{3}\left(t_{k}^{+}\right)}, \hat{q}_{3}\left(t_{k+1}\right)\right\rangle \\
& >\frac{\widetilde{m}_{2}\left(t_{k}^{+}\right)}{\widetilde{m}_{3}\left(t_{k}^{+}\right)}\left\langle\tilde{p}_{3}\left(t_{k}^{+}\right), \hat{q}_{3}\left(t_{k+1}\right)\right\rangle>\frac{\widetilde{m}_{2}\left(t_{k}^{+}\right)}{\widetilde{m}_{3}\left(t_{k}^{+}\right)}\left\langle\tilde{p}_{3}\left(t_{k}^{+}\right), \hat{q}_{3}\left(t_{k}\right)\right\rangle>0 .
\end{aligned}
$$


III. Finally we use (3.18) to show that

$$
\begin{aligned}
D_{3, I I I}(k) & =\widetilde{m}_{2}\left(t_{k+1}^{+}\right)\left\langle\frac{\tilde{q}_{3}\left(t_{k+1}\right)-\tilde{q}_{1}\left(t_{k+1}\right)}{t_{k+2}-t_{k+1}}-\frac{\tilde{p}_{1}\left(t_{k+1}^{+}\right)}{\widetilde{m}_{1}\left(t_{k+1}^{+}\right)}, \hat{q}_{3}\left(t_{k+1}\right)\right\rangle \\
& >-\frac{\widetilde{m}_{2}\left(t_{k+1}^{+}\right)}{\widetilde{m}_{1}\left(t_{k+1}^{+}\right)}\left\langle\tilde{p}_{1}\left(t_{k+1}^{+}\right), \hat{q}_{3}\left(t_{k+1}\right)\right\rangle \\
& =\frac{\widetilde{m}_{2}\left(t_{k+1}^{+}\right)}{\widetilde{m}_{1}\left(t_{k+1}^{+}\right)}\left\langle\tilde{p}_{1}\left(t_{k}^{+}\right), \hat{q}_{1}\left(t_{k+1}\right)\right\rangle \\
& \geq \frac{\widetilde{m}_{2}\left(t_{k+1}^{+}\right)}{\widetilde{m}_{1}\left(t_{k+1}^{+}\right)}\left\langle\tilde{p}_{1}\left(t_{k}^{+}\right), \hat{q}_{1}\left(t_{k}\right)\right\rangle>0 .
\end{aligned}
$$

Thus (3.22) is estimated from below, with $\tilde{K}_{\|}$from (3.19), by

$$
\begin{aligned}
C_{3}(k) & >\frac{\left(D_{3, I I}(k)\right)^{2}+\left(D_{3, I I I}(k)\right)^{2}}{2 M} \\
& >\frac{\left(\frac{\widetilde{m}_{2}\left(t_{k}^{+}\right)}{\tilde{m}_{3}\left(t_{k}^{+}\right)}\left\langle\tilde{p}_{3}\left(t_{k}^{+}\right), \hat{q}_{3}\left(t_{k}\right)\right\rangle\right)^{2}+\left(\frac{\tilde{m}_{2}\left(t_{k+1}^{+}\right)}{\tilde{m}_{1}\left(t_{k+1}^{+}\right)}\left\langle\tilde{p}_{1}\left(t_{k}^{+}\right), \hat{q}_{1}\left(t_{k}\right)\right\rangle\right)^{2}}{2 M} \\
& \geq\left(\frac{m_{\min }}{m_{\max }}\right)^{2} \tilde{K}_{\|}\left(t_{k}^{+}\right) .
\end{aligned}
$$

Together, (a) and (b) prove (3.20), and thus (3.9) follows.

5. The alignment of the directions $\hat{v}_{i}$ follows from the above statements, using the bounds on the angular momenta:

There is a unique closed circular segment $\operatorname{seg}\left(\hat{v}_{2}\left(t_{k+1}^{-}\right), \hat{v}_{3}\left(t_{k+1}^{-}\right)\right) \subseteq S^{1}$ of length in $[0, \pi)$ between $\hat{v}_{2}\left(t_{k+1}^{-}\right)$and $\hat{v}_{3}\left(t_{k+1}^{-}\right)$. Still the oriented lines $\ell_{1}(k)$ and $\ell_{3}(k)$ may be antiparallel, so that $\hat{v}_{1}\left(t_{k+1}^{-}\right)=-\hat{v}_{3}\left(t_{k+1}^{-}\right)$. One concludes from $t_{k+1} \in\left(t_{k},+\infty\right)$ that for $k$ odd

$$
-\hat{v}_{2}\left(t_{k+1}^{+}\right) \in \begin{cases}\operatorname{seg}\left(-\hat{v}_{1}\left(t_{k+1}\right), \hat{v}_{2}\left(t_{k+1}^{-}\right)\right) & , k \text { odd } \\ \operatorname{seg}\left(-\hat{v}_{3}\left(t_{k+1}\right), \hat{v}_{2}\left(t_{k+1}^{-}\right)\right) & , k \text { even }\end{cases}
$$

see Figure 3.1. The subsets

$$
S_{k}:=\left\{\begin{array}{l}
\operatorname{seg}\left(-\hat{v}_{1}\left(t_{k+1}\right), \hat{v}_{2}\left(t_{k+1}^{-}\right)\right) \cup \operatorname{seg}\left(\hat{v}_{2}\left(t_{k+1}^{-}\right), \hat{v}_{3}\left(t_{k+1}^{-}\right)\right), k \text { odd } \\
\operatorname{seg}\left(-\hat{v}_{3}\left(t_{k+1}\right), \hat{v}_{2}\left(t_{k+1}^{-}\right)\right) \cup \operatorname{seg}\left(\hat{v}_{2}\left(t_{k+1}^{-}\right), \hat{v}_{1}\left(t_{k+1}^{-}\right)\right), k \text { even }
\end{array}\right.
$$

of $S^{1}$ are segments, since both segments on the r.h.s. of (3.24) contain the end point $\hat{v}_{2}\left(t_{k+1}^{-}\right)$.

From the preservation of total momentum at time $t_{k+1}$, that is (3.21), it follows that

$$
S_{k+2} \subseteq S_{k} \quad(k \in \mathbb{N}) .
$$

Moreover, we show now that the lengths of the segments $S_{k}$ converge to zero exponentially in $k$ : 
- At the times $t_{k}$ of collision, in the center of mass frame, all three particles are on a line through the origin, and from (3.7), exponentially in $k$

$$
\lim _{k \rightarrow \infty}\left\|\tilde{q}_{i}\left(t_{k}\right)\right\|=\infty \quad(i=1,2,3) .
$$

- By (3.9) the last statement is also true for the speeds: Exponentially in $k$

$$
\lim _{k \rightarrow \infty}\left\|\tilde{v}_{i}^{ \pm}\left(t_{k}\right)\right\|=\infty \quad(i=1,2,3) .
$$

- On the other hand, the angular momenta are uniformly bounded by (3.5).

These three statements are only compatible, if the directions $\hat{v}_{i}\left(t_{k}^{ \pm}\right) \in S^{1}$ $(i=1,2,3)$ become (anti-) parallel as $k \rightarrow \infty$. Together with (3.25), we conclude that, as $k \rightarrow \infty$, there is a limiting line they span.
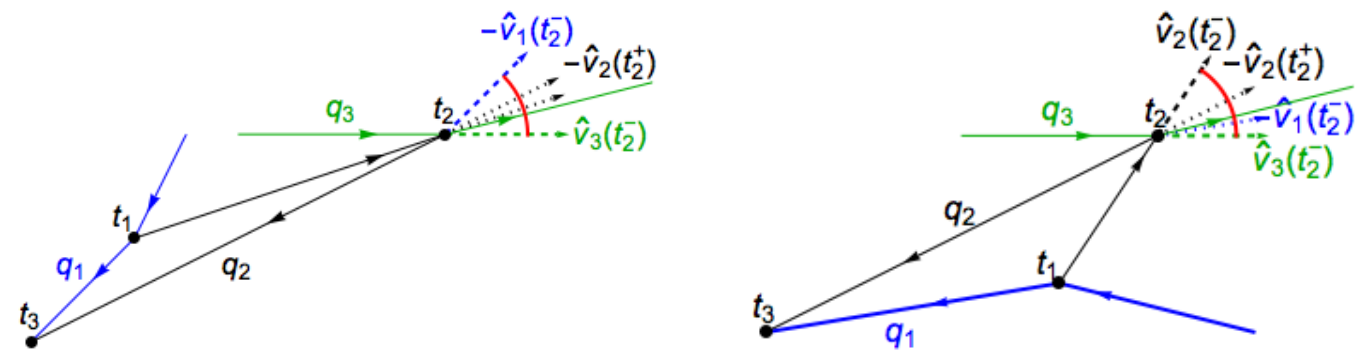

Figure 3.1: Alignment of velocities, here of particle 2 and 3 at time $t_{k+1} \equiv t_{2}$. The segment $S_{k}$ containing $\hat{v}_{3}\left(t_{2}^{+}\right)$is shown in red. Note the different orientations in the two pictures, and that the third possibility $S_{k}=\operatorname{seg}\left(-\hat{v}_{1}\left(t_{k+1}^{-}\right), \hat{v}_{2}\left(t_{k+1}^{-}\right)\right)$ is also realizable.

\section{Dynamics}

The aim of this section is to provide the dynamical estimates that will be used to show, in Section 5.2, that for every total energy $E \in \mathbb{R}$, with the $\mathfrak{L}$-dependent family of Poincaré sections $\mathcal{H}_{m, E, \mathfrak{L}} \subseteq \Sigma_{E, 0}$ defined in (5.7) and (5.11), and

$$
\begin{gathered}
\operatorname{Trans}_{E, 0, \mathfrak{L}}:=\left\{x \in \Sigma_{E, 0} \mid \exists m_{0} \in \mathbb{N} \forall m \geq m_{0}: \mathcal{O}^{+}(x) \cap \overline{\mathcal{H}}_{m, E, \mathfrak{L}} \neq \emptyset\right\} \\
\operatorname{NAV}_{E, 0} \subseteq \bigcup_{\mathfrak{L}>0} \operatorname{Trans}_{E, 0, \mathfrak{L}} .
\end{gathered}
$$


In words: in the center of mass frame, for every initial condition $x$ of energy $E$ whose asymptotic velocity limit (1.3) does not exist, there is an angular momentum parameter $\mathfrak{L}$, such that its forward orbit intersects almost all Poincaré surfaces $\mathcal{H}_{m, E, \mathfrak{L}}$ of that parameter.

Considering the definition (5.11) of the Poincare surfaces, the main task will be to control the evolution of the cluster angular momenta. Total angular momentum $L$, see (2.13), is a constant of the motion. As shown in Lemma 5.1 (based on estimates of the current section) $\|L(x)\|$ sets a scale for the angular momenta of the clusters, and thus for the parameter $\mathfrak{L}$ for which $x \in \operatorname{Trans}_{E, 0, \mathfrak{L}}$.

It has been proven in Section 2.5 that for initial conditions $x \in \mathrm{NAV}$ and times $t \in\left[t_{0}, T(x)\right)$ the cluster size $|\mathcal{A}(t)|$ is two or three, and that the time interval is partitioned into subintervals $\left(t_{k}, t_{k+1}\right)$ where for $k$ even, $\mathcal{A}$ is constant and of size $\left|\mathcal{A}\left(t_{k}^{+}\right)\right|=3$, whereas $\left|\mathcal{A}\left(t_{k}^{-}\right)\right|=\left|\mathcal{A}\left(t_{k+1}^{+}\right)\right|=2$ and $\mathcal{A}\left(t_{k}^{-}\right)$and $\mathcal{A}\left(t_{k+1}^{+}\right)$are not comparable. Accordingly, we have to tackle two dynamical problems:

- For $k$ odd, the internal motion of the cluster $C_{1} \cup C_{2}$, perturbed by cluster $C_{3}$. Then the relative cluster angular momentum (4.3) of the pair $C_{1}, C_{2}$ of clusters is small, see Section 4.2.

- For $k$ even and $\mathcal{A}(t)=\left\{C_{1}, C_{2}, C_{3}\right\}$, the flight of the messenger cluster $C_{2}$ from $C_{1}$ to $C_{3}$. The cluster centers will asymptotically move on straight lines, see Section 4.3.

\subsection{Preparatory estimates}

To obtain the dynamical estimates, we use:

1. By the von Zeipel Theorem 2.6, the limit $j^{+}(x):=\lim _{t} \nearrow_{T^{+}(x)} j_{x}(t)$ exists and equals $+\infty$. Additionally, inspection of (5.5) shows that for all $t \in\left(t_{k}, t_{k+1}\right)$ the time derivative $\frac{d}{d t} \tilde{J}_{\mathcal{A}}^{E}$ of the external moment of inertia is positive and goes to infinity. For $k$ odd this means that both $C_{1} \cup C_{2}$ and $C_{3}$ move away from the origin, with opposite momenta (in the center of mass frame).

2. When $t$ is a 'messenger time', i.e., $t \in$ mess with

$$
\text { mess }:=\cup_{k \in 2 \mathbb{N}}\left(t_{k}, t_{k+1}\right),
$$

we have $|\mathcal{A}(t)|=3$ clusters, and the messenger cluster $C_{2}$, see above. This implies for the unique nontrivial cluster $D(t) \in \mathcal{A}(t), t \in$ mess, that

4.1 Lemma (The nontrivial cluster) For $x \in \mathrm{NAV}$ and $E:=H(x)$,

(a) The external potential energy $\tilde{V}_{\mathcal{A}(t)}^{E}(t)=o(1) \quad(t \nearrow T(x))$, and $\tilde{V}_{\mathcal{A}(t)}^{I}(t)=\tilde{V}_{D(t)}^{I}(t)$ for $t \in$ mess. 
(b) The external cluster energy has the limit $\lim _{t \nearrow T(x)} \tilde{H}_{\mathcal{A}(t)}^{E}(t)=+\infty$.

(c) The internal energy of the unique nontrivial cluster $D(t)$ has the limit

$$
\lim _{t \succ T(x)} \tilde{H}_{D(t)}^{I}(t)=-\infty
$$

(d) The internal angular momentum (see (2.15)) of $D(t)$ has the limit

$$
\lim _{\substack{t T(x) \\ t \in \operatorname{mess}}} \tilde{L}_{D(t)}^{I}=0
$$

\section{Proof:}

(a) The first property is valid for all initial conditions $x \in P$, as in $\mathcal{A}(t)$ (see (5.4)) the different cluster centers have distances bounded below by $\delta^{2} t^{1-\varepsilon / 2}$. The identity $\tilde{V}_{\mathcal{A}(t)}^{I}(t)=\tilde{V}_{D(t)}^{I}(t)$ follows from the Definition (2.16) of $V_{\mathcal{C}}^{I}$, since both clusters in $\mathcal{A}(t) \backslash\{C(t)\}$ are trivial.

(b) By Theorem 2.8.1, $\lim _{t \nearrow T(x)}\left\|\frac{d}{d t} q_{\mathcal{A}(t)}^{E}(t)\right\|_{\mathcal{M}}=\infty$ for the cluster-external speed. So the total kinetic cluster energy diverges: $\lim _{t \nearrow T(x)} \tilde{K}_{\mathcal{A}(t)}^{E}(t)=+\infty$. Since $\tilde{H}_{\mathcal{A}}^{E}=\tilde{K}_{\mathcal{A}}^{E}+\tilde{V}_{\mathcal{A}}^{E}$ (see (2.17)), (b) follows from Part (a).

(c) follows from Part (b) by energy conservation, as $E=\tilde{H}=\tilde{H}_{\mathcal{A}}^{E}+\tilde{H}_{\mathcal{A}}^{I}$, and for $t \in \operatorname{mess}, \tilde{H}_{\mathcal{A}(t)}^{I}(t)=\tilde{H}_{D(t)}^{I}(t)$.

(d) As $H_{D}^{I}=K_{D}^{I}+V_{D}^{I}$, with $K_{D}^{I}\left(p_{D}^{I}, q_{D}^{I}\right) \geq \frac{\left\|L_{D}^{I}\right\|^{2}}{2 m_{D}^{I}\left\|q_{D}^{I}\right\|^{2}}$ and $V_{D}^{I}\left(q_{D}^{I}\right) \geq-\frac{I}{\left\|q_{D}^{I}\right\|^{\alpha}}$ with $\alpha<2$, Statement (c) is only possible if Assertion (d) is true.

For the three-tuple $\left(C_{1}, C_{2}, C_{3}\right) \in \mathcal{T}$ with $\mathcal{A}(t)=\left\{C_{1}, C_{2}, C_{3}\right\}$, attributed to the interval $\left(t_{k}, t_{k+1}\right)$ (see page 11 ), the messenger cluster $C_{2}$ carries a positive proportion of the total kinetic energy $\tilde{K}_{\mathcal{A}}^{E}=\sum_{i=1}^{3} \tilde{K}_{C_{i}}^{E}$ :

\subsection{Lemma (Kinetic energy of the messenger cluster)}

$$
\tilde{K}_{C_{2}(t)}^{E}(t) \geq \frac{m_{\min }}{7 m_{\max }} \tilde{K}_{\mathcal{A}(t)}^{E}(t) \quad(t \in \text { mess }) .
$$

Proof: This follows for a certain $t \in\left(t_{k}, t_{k+1}\right)$, since the speed $\left\|v_{C_{2}}\right\|$ of the messenger particle must be at least the one of the cluster $C_{3}$ it is to reach, and

$$
\begin{aligned}
2 \tilde{K}_{\mathcal{A}}^{E} & =m_{C_{1}}\left\|\tilde{v}_{C_{2}}+\tilde{v}_{C_{3}}\right\|^{2}+m_{C_{2}}\left\|\tilde{v}_{C_{2}}\right\|^{2}+m_{C_{3}}\left\|\tilde{v}_{C_{3}}\right\|^{2} \\
& \leq m_{C_{1}}\left\|2 \tilde{v}_{C_{2}}\right\|^{2}+m_{C_{2}}\left\|\tilde{v}_{C_{2}}\right\|^{2}+m_{C_{3}}\left\|\tilde{v}_{C_{2}}\right\|^{2} \leq 6 m_{\max }\left\|\tilde{v}_{C_{2}}\right\|^{2} \leq \frac{6 m_{\max }}{m_{\min }} 2 \tilde{K}_{C_{2}}^{E} .
\end{aligned}
$$


It will follow for all $t \in\left(t_{k}, t_{k+1}\right)$ (with factor 7 instead of 6 ) from the propagation estimates of Sect. 4.3, since the velocities $\tilde{v}_{C_{2}}$ are nearly constant in $\left(t_{k}, t_{k+1}\right)$.

Thus, when at time $t_{k}$ cluster $C_{2}$ separates from cluster $C_{1}$, their relative kinetic energy is not negligible, compared to the internal energy of the cluster $D$. This fact will be used in Lemma 4.5, concerning the motion of the messenger cluster. Although the scheme $\ldots \rightarrow \mathcal{A}\left(t_{k}\right) \rightarrow \mathcal{A}\left(t_{k+1}\right) \rightarrow \ldots$, depicted in (4.2),

$$
\left\{C_{1}^{\prime}, C_{2}^{\prime} \cup C_{3}\right\} \rightarrow\left\{C_{1}^{\prime}, C_{2}^{\prime}, C_{3}\right\} \rightarrow\left\{C_{1,2}, C_{3}\right\} \rightarrow\left\{C_{1}, C_{2}, C_{3}\right\} \rightarrow\left\{C_{1}, C_{2} \cup C_{3}\right\}
$$

repeats after two steps, when showing in Section 4.2 that the relative angular momentum of $C_{1}$ and $C_{2}$ must be small we use that in both time directions the messenger cluster $C_{2}$ resp. $C_{2}^{\prime}$ returns from $C_{1,2}:=C_{1} \cup C_{2}=C_{1}^{\prime} \cup C_{2}^{\prime}$ to $C_{3}$.

As Sections 4.2 and 4.3 consider the situation where the cluster centers of $C_{2}$ and $C_{1}$ (or $C_{3}$ ) are initially very close, we now introduce the quantities that are adapted to that situation.

For two mutually disjoint clusters $C, D \subseteq N$, the following quantities neither depend on the reference frame nor on the ordering of $C$ and $D$ : total mass $m_{C \cup D}=m_{C}+m_{D}$, reduced mass $m_{C, D}:=\frac{m_{C} m_{D}}{m_{C}+m_{D}}$, and the relative phase space functions

$$
\begin{aligned}
L_{C, D} & :=\frac{1}{2}\left(q_{C}-q_{D}\right) \wedge\left(p_{C}-p_{D}\right) \\
K_{C, D} & :=\frac{1}{2} m_{C, D}\left\|v_{C}-v_{D}\right\|^{2} \\
H_{C, D} & :=K_{C, D}+V_{C, D} \quad \text { with } \quad V_{C, D}:=\sum_{i \in C, j \in D} V_{i, j} \\
J_{C, D}(q) & :=\frac{1}{2}\left(m_{C}\left\|q_{C}\right\|^{2}+m_{D}\left\|q_{D}\right\|^{2}\right) .
\end{aligned}
$$

\subsection{Near-collision of two clusters}

We now consider the near-collision of the clusters $C_{1}$ and $C_{2}$ that took place before time $s_{1}$, see (4.2).

For simplicity of presentation, with the involution $\mathcal{R}:(p, q) \mapsto(-p, q)$ we reverse the direction of time. That does not change the various kinetic energies and reverses the sign of the angular momenta. W.I.o.g. we set $s_{1}:=0$.

If initially (at time 0 ) the cluster $D \in\left\{C_{1}, C_{2}, C_{3}\right\}$ that is nontrivial, equals $C_{1}$ or $C_{2}$, a close encounter of the three bodies $C_{1,2}=C_{1} \cup C_{2}$ follows.

Otherwise $D=C_{3}$, and the close encounter involves only two bodies.

Since the initial condition is $x \in \mathrm{NAV}_{E}$, we can assume that

1. $|E| \ll-\tilde{H}_{D}^{I}\left(s_{1}\right)$, as $\tilde{H}_{D}^{I}(t) \searrow-\infty$ by Lemma 4.1 (c).

2. The relative kinetic energy $K_{C_{1}, C_{2}}$ of the two clusters (see (4.4)) is large:

$$
\tilde{K}_{C_{1}, C_{2}}(0) \geq \frac{m_{\min }}{7 m_{\max }}\left(-\tilde{H}_{D}^{I}(0)\right) \text {. }
$$


That assumption is justified, as by Lemma 4.2 on the partition of kinetic energy $\tilde{K}_{C_{2}}^{E} \geq \frac{m_{\min }}{7 m_{\max }} \tilde{K}_{\mathcal{A}}^{E}$.

3. Repeating the notation from (4.2), after having reversed the direction of time,

(a) in the past $C_{2}$ had a near-collision with $C_{3}$

(b) after the near-collision between $C_{1}$ and $C_{2}, C_{1,2}$ decomposes into $C_{1}^{\prime}$ and $C_{2}^{\prime}\left(C_{1,2}=C_{1}^{\prime} \cup C_{2}^{\prime}\right)$,

(c) finally $C_{2}^{\prime}$ has a near-collision with $C_{3}^{\prime}=C_{3}$.

As by the propagation estimates between near-collision the cluster centers have nearly constant velocities, this means that the direction of $p_{C_{2}^{\prime}}$ must be nearly opposite to the one of $p_{C_{2}}$. We show that this can only be the case if initially the norm of the relative angular momentum $L_{C_{1}, C_{2}}$, see (4.3), of $C_{1}$ and $C_{2}$ has small modulus. The basic estimate is Statement 3 of Lemma 4.3.

First, in Lemma 4.3, we consider the case of no interaction between the two clusters $C_{1,2}$ and $C_{3}: V_{\left\{C_{1,2}, C_{3}\right\}}^{E}=0$, see (2.16) and Assumption 3 below. Then the centers of mass $q_{C_{1,2}}$ and $q_{C_{3}}$ move with constant velocities.

So we change from the center of mass frame of $C_{N}$ to the one of $C_{1,2}$, i.e.

$$
\sum_{i \in C_{1,2}} p_{i}=0 \quad, \quad \sum_{i \in C_{1,2}} m_{i} q_{i}=0
$$

without modifying the notation. By Assumption $3, H_{C_{1,2}}^{I}$ is a constant of the motion, and its Hamiltonian equations describe the relative motion of the single particles $C_{1}$ and $C_{2}$. If $D \neq C_{3}$,

$$
H_{C_{1,2}}^{I}=H_{C_{1}, C_{2}}+H_{D}^{I} \quad \text { for } \quad H_{C_{1}, C_{2}}=K_{C_{1}, C_{2}}+V_{C_{1}, C_{2}},
$$

see (4.5). For $D=C_{3}, H_{C_{1,2}}^{I}=H_{C_{1}, C_{2}}$, and $H_{D}^{I}$ is a constant of the motion. The total angular momentum $L_{C_{1,2}}=\sum_{i=1}^{3} q_{i} \wedge p_{i}$ of $C_{1,2}$ is a constant of motion, too.

When we treat the case $D=C_{1}$ (or equivalently $D=C_{2}$ ), by permuting indices, if necessary, we assume $C_{1}=D=\{1,2\}, C_{2}=\{3\}$ and $C_{3}=\{4\}$. We then use cluster coordinates for $D$, i.e. apply the linear symplectomorphism $\Psi: T^{*} \mathbb{R}^{4 d} \rightarrow T^{*} \mathbb{R}^{4 d}$ of the phase space, mapping $\left(p_{1}, p_{2}, q_{1}, q_{2}\right)$ to

$$
\left(p_{D}, p_{D}^{I}, q_{D}, q_{D}^{I}\right):=\left(p_{1}+p_{2}, \frac{m_{2} p_{1}-m_{1} p_{2}}{m_{D}}, \frac{m_{1} q_{1}+m_{2} q_{2}}{m_{D}}, q_{1}-q_{2}\right)
$$

with cluster mass $m_{D}=m_{1}+m_{2}$ and reduced mass $m_{D}^{I}=\frac{m_{1} m_{2}}{m_{1}+m_{2}}$ of $D$, and preserving the other coordinates. In the $C_{1,2}$ center of mass frame, see (4.7)

$$
p_{3}=-p_{D} \quad \text { and } \quad q_{3}=-\frac{m_{D}}{m_{3}} q_{D}
$$


We do not rename phase space functions, transformed with $\Psi$. So in (4.8)

$$
K_{C_{1}, C_{2}}=\frac{\left\|p_{D}\right\|^{2}}{2 m_{C_{1}, C_{2}}} \quad, \quad H_{D}^{I}=\frac{\left\|p_{D}^{I}\right\|^{2}}{2 m_{D}^{I}}+V_{1,2}\left(q_{D}^{I}\right)
$$

(whereas $K_{D}=\frac{\left\|p_{D}\right\|^{2}}{2 m_{D}}$ ) and $^{2}$

$$
V_{C_{1}, C_{2}}=V_{1,3}\left(\frac{m_{C_{1,2}}}{m_{3}} q_{D}+\frac{m_{2}}{m_{D}} q_{D}^{I}\right)+V_{2,3}\left(\frac{m_{C_{1,2}}}{m_{3}} q_{D}-\frac{m_{1}}{m_{D}} q_{D}^{I}\right) .
$$

Relative angular momentum $L_{C_{1}, C_{2}}$ from (4.3) is a term of $L_{C_{1,2}}=\frac{1}{2} \sum_{k=1}^{3} q_{k} \wedge p_{k}$ : $L_{C_{1,2}}=L_{C_{1}, C_{2}}+L_{D}^{I} \quad$ with $L_{C_{1}, C_{2}}=\frac{m_{D}}{m_{C_{1}, C_{2}}} q_{D} \wedge p_{D}$ and $L_{D}^{I}=q_{D}^{I} \wedge p_{D}^{I}$.

Similarly, $J_{C_{1,2}}=\frac{1}{2} \sum_{k=1}^{3} m_{k}\left\|q_{k}\right\|^{2}$ and the relative moment of inertia (4.6) are related by

$$
J_{C_{1,2}}=J_{C_{1}, C_{2}}+J_{D}^{I} \quad \text { with } J_{C_{1}, C_{2}}=\frac{m_{D}^{2}}{2 m_{C_{1}, C_{2}}}\left\|q_{D}\right\|^{2} \text { and } J_{D}^{I}=\frac{1}{2} m_{D}^{I}\left\|q_{D}^{I}\right\|^{2} \text {. }
$$

Assumption 1 in the following lemma is eventually satisfied by all orbits, for which asymptotic velocity does not exist. Assumption 2 is satisfied for no such orbit. After showing in Lemma 4.4 that Assumption 3 can essentially be skipped, this will give us an upper bound on the cluster angular momentum of such orbits.

\subsection{Lemma (Near-collision of $C_{1}$ and $C_{2}$, no interaction with $C_{3}$ )}

Consider for initial condition $\tilde{x}(0) \in \mathrm{NAV}_{E, 0}$ the motion $t \mapsto \tilde{x}(t)=(\tilde{p}(t), \tilde{q}(t))$ on $\Sigma_{E, 0}$, whose initial condition fulfills the following assumptions:

1. For some $\delta \in(0,1 / 2)$, initially the energies are related by (see (4.5))

$$
|E| \leq-\delta \tilde{H}_{D}^{I}(0)
$$

2. For the relative cluster angular momentum (4.3) (with I from Remark 1.2),

$$
\left\|\tilde{L}_{C_{1}, C_{2}}(0)\right\| \geq C \tilde{K}_{C_{1}, C_{2}}(0)^{-\frac{2-\alpha}{2 \alpha}} I^{1 / \alpha}\left(m_{C_{1}, C_{2}}\right)^{1 / 2} .
$$

3. There is no interaction between the clusters $C_{1,2}$ and $C_{3}$ :

$$
V_{\left\{C_{1,2}, C_{3}\right\}}^{E}(q) \equiv \sum_{i \in C_{1,2}, j \in C_{3}} V_{i, j}\left(q_{i}-q_{j}\right)=0 \quad(q \in \widehat{M}) .
$$

Then for $C$ in (4.14) large, one has, for times in $\left\{t \in \mathbb{R} \mid\left\|\tilde{q}_{D}(t)\right\| \leq 1\right\}$ :

$2 \frac{m_{C_{1,2}}}{m_{3}}=\frac{m_{D}}{m_{C_{1}, C_{2}}}>1$ is the ratio of the distance of the cluster centers and of $\left\|q_{D}\right\|$. 
1. The variation of relative kinetic energy and cluster angular momentum is small:

$$
\begin{aligned}
\left|\tilde{K}_{C_{1}, C_{2}}(t)-\tilde{K}_{C_{1}, C_{2}}(0)\right| & =\mathcal{O}\left(C^{-\alpha}\right) \tilde{K}_{C_{1}, C_{2}}(0), \\
\left\|\tilde{L}_{C_{1}, C_{2}}(t)-\tilde{L}_{C_{1}, C_{2}}(0)\right\| & =\mathcal{O}\left(C^{-\alpha}\right)\left\|\tilde{L}_{C_{1}, C_{2}}(0)\right\|,
\end{aligned}
$$

and

$$
-(1+2 \delta) \tilde{H}_{D}^{I}(t) \geq \tilde{K}_{C_{1}, C_{2}}(t)
$$

2. $\left\|\tilde{q}_{D}^{I}\right\|<\left(\frac{2 I}{\tilde{K}_{C_{1}, C_{2}}}\right)^{1 / \alpha}$, but $\left\|\tilde{q}_{D}\right\| \geq C \frac{m_{C_{1}, C_{2}}}{2 m_{D}}\left(\frac{I}{\tilde{K}_{C_{1}, C_{2}}}\right)^{1 / \alpha}$, thus $\left\|\tilde{q}_{D}^{I}\right\| \ll\left\|\tilde{q}_{D}\right\|$.

3. The total change of direction $\frac{\tilde{q}_{C_{i}}^{\prime}(t)}{\left\|\tilde{q}_{C_{i}}^{\prime}(t)\right\|}$ of the cluster centers is of order

$$
\int_{\mathbb{R}} \frac{\left\|\dot{\tilde{q}}_{C_{i}} \wedge \ddot{\tilde{q}}_{C_{i}}\right\|}{\left\|\tilde{\tilde{q}}_{C_{i}}\right\|^{2}} d t=\mathcal{O}\left(C^{-\alpha}\right) \quad(i=1,2) .
$$

Proof: The proof will be devious, as we first assume statements concerning $\tilde{K}_{C_{1}, C_{2}}, \tilde{L}_{C_{1}, C_{2}}$ and $\tilde{H}_{D}^{I}$ that are weaker than the ones of Assertion 1 (see (4.20$4.21)$ ), then conditionally show all assertions of the lemma, and thus prove (in Parts 3, 7 and 8) that these a priori inequalites (valid for $t=0$ ) were justified.

We only consider the three-body case $\left|C_{1,2}\right|=3$, since the simpler two-body case $\left|C_{1,2}\right|=2$ leads to even better estimates. Then the nontrivial cluster $D$ equals $C_{1}$ (or, equivalently, $C_{2}$ ). By Remark 1.2, Estimate (1.4) is applicable to the pair potentials $V_{i, j}$ for times $t$ with $\left\|\tilde{q}_{D}(t)\right\| \leq 1$.

We henceforth assume the a priori inequalities for all $t$ in $\left\{t \in \mathbb{R} \mid\left\|\tilde{q}_{D}(t)\right\| \leq 1\right\}$ :

$$
\begin{aligned}
\left|\tilde{K}_{C_{1}, C_{2}}(t)-\tilde{K}_{C_{1}, C_{2}}(0)\right| & \leq \frac{1}{2} \tilde{K}_{C_{1}, C_{2}}(0) \\
\left\|\tilde{L}_{C_{1}, C_{2}}(t)\right\| & \geq \frac{1}{2} C \tilde{K}_{C_{1}, C_{2}}(t)^{-\frac{2-\alpha}{2 \alpha}} I^{1 / \alpha} \sqrt{m_{C_{1}, C_{2}}} \\
-2 \tilde{H}_{D}^{I}(t) & \geq \tilde{K}_{C_{1}, C_{2}}(t) .
\end{aligned}
$$

The one for $\tilde{L}_{C_{1}, C_{2}}(t)$ is similar to (4.14) for $t=0$. For $C$ large, these are weaker than the assertions (4.15), (4.16) and (4.17).

1. By (4.11) in the $C_{1,2}$ center of mass frame $L_{C_{1}, C_{2}}=\frac{m_{D}}{m_{C_{1}, C_{2}}} q_{D} \wedge p_{D}$, so that by (4.9) and the a priori inequality (4.20), with $c_{1}:=\frac{m_{C_{1}, C_{2}}}{2 m_{D}}$,

$$
\left\|\tilde{q}_{D}\right\| \geq \frac{m_{C_{1}, C_{2}}}{m_{D}} \frac{\left\|\tilde{L}_{C_{1}, C_{2}}\right\|}{\left\|\tilde{p}_{D}\right\|}=\frac{\left\|\tilde{L}_{C_{1}, C_{2}}\right\|}{m_{D}}\left(\frac{m_{C_{1}, C_{2}}}{2 \tilde{K}_{C_{1}, C_{2}}}\right)^{1 / 2} \geq c_{1} C\left(I / \tilde{K}_{C_{1}, C_{2}}\right)^{1 / \alpha}
$$

So conditionally on (4.20), we have shown the estimate of $\tilde{q}_{D}$ in Statement 2. 
2. The estimate on $\tilde{q}_{D}^{I}$ in Statement 2. follows from the a priori inequality (4.21):

$$
\left\|\tilde{q}_{D}^{I}\right\| \leq\left(\frac{I}{\left|\tilde{V}_{1,2}\right|}\right)^{1 / \alpha} \leq\left(\frac{I}{\left|\tilde{H}_{D}^{I}\right|}\right)^{1 / \alpha} \leq\left(\frac{2 I}{\tilde{K}_{C_{1}, C_{2}}}\right)^{1 / \alpha} .
$$

So (4.21) implies for $C$ large that the relative cluster energy $\tilde{H}_{C_{1}, C_{2}}(t)$ is mainly kinetic in the following sense:

$$
0<I\left\|\tilde{q}_{D}\right\|^{-\alpha} \leq c_{1}^{-\alpha} C^{-\alpha} \tilde{K}_{C_{1}, C_{2}} .
$$

In particular, for $c_{2}:=3 c_{1}^{-\alpha}$ and $C$ large,

$$
\left|\tilde{V}_{C_{1}, C_{2}}\right| \leq c_{2} C^{-\alpha} \tilde{K}_{C_{1}, C_{2}} \quad \text { and } \quad\left|\left\langle q_{D}, \nabla_{q_{D}} \tilde{V}_{C_{1}, C_{2}}\right\rangle\right| \leq c_{2} C^{-\alpha} \tilde{K}_{C_{1}, C_{2}} .
$$

3. As by Assumptions 1. and 3., the constant $\tilde{H}_{C_{1,2}}^{I}$ is bounded from above by

$$
\tilde{H}_{C_{1,2}}^{I}=E-\tilde{H}_{\left\{C_{1,2}, C_{3}\right\}}=E-\tilde{K}_{\left\{C_{1,2}, C_{3}\right\}} \leq E \leq-\delta \tilde{H}_{D}^{I}(0),
$$

and by (4.25) and (4.8),

$$
0<\left(1-c_{2} C^{-\alpha}\right) \tilde{K}_{C_{1}, C_{2}} \leq \tilde{H}_{C_{1}, C_{2}}=\tilde{H}_{C_{1,2}}^{I}-\tilde{H}_{D}^{I} \leq-(1+\delta) \tilde{H}_{D}^{I}
$$

So $-(1+2 \delta) \tilde{H}_{D}^{I} \geq \tilde{K}_{C_{1}, C_{2}}$ for $C$ large, showing (4.17) conditionally on (4.20-4.21).

4. To control the time evolution of $q_{D}$, we consider $\tilde{J}_{C_{1}, C_{2}}$ in the $C_{1,2}$-center of mass frame, see (4.12). Then

$$
\frac{d^{2}}{d t^{2}} \tilde{J}_{C_{1}, C_{2}}=2 \tilde{K}_{C_{1}, C_{2}}+\left\langle\tilde{q}_{D}, \nabla_{q_{D}} V_{C_{1}, C_{2}}(\tilde{q})\right\rangle
$$

Then with (4.25), for $C$ large (4.26) leads to the inequality

$$
\frac{d^{2}}{d t^{2}} \tilde{J}_{C_{1}, C_{2}} \geq \tilde{K}_{C_{1}, C_{2}} .
$$

5. So with the a priori estimate (4.19) (implying $\tilde{K}_{C_{1}, C_{2}}(t) \geq \frac{1}{2} \tilde{K}_{C_{1}, C_{2}}(0)>0$ ),

$$
\frac{d^{2}}{d t^{2}} \tilde{J}_{C_{1}, C_{2}} \geq \frac{1}{2} \tilde{K}_{C_{1}, C_{2}}(0)>0 .
$$

Thus there is a unique time $t_{0}$ where $\tilde{J}_{C_{1}, C_{2}}$ attains its minimum, and by (4.12), (4.22) and (4.19) we get the propagation estimate

$$
\begin{aligned}
\tilde{J}_{C_{1}, C_{2}}(t) & \geq \tilde{J}_{C_{1}, C_{2}}\left(t_{0}\right)+\frac{1}{4} \tilde{K}_{C_{1}, C_{2}}(0)\left(t-t_{0}\right)^{2} \\
& \left.\geq \frac{1}{8} m_{C_{1}, C_{2}} C^{2}\left(\frac{2}{3} I / \tilde{K}_{C_{1}, C_{2}}(0)\right)\right)^{\frac{2}{\alpha}}+\frac{1}{4} \tilde{K}_{C_{1}, C_{2}}(0)\left(t-t_{0}\right)^{2} .
\end{aligned}
$$

So in both time directions the distance between the cluster centers $q_{C_{1}}$ and $q_{C_{2}}$ diverges (at least) linearly. 
6. We now show Statement 3., estimating $\int_{\mathbb{R}}\left(\tilde{J}_{C_{1}, C_{2}}\right)^{-(1+\alpha) / 2} d t$ by use of $(4.27)$ and with

$$
\int_{\mathbb{R}}\left(b+c t^{2}\right)^{-(1+\alpha) / 2} d t=\frac{C_{1}(\alpha)}{b^{\alpha / 2} \sqrt{c}} \quad \text { for } \quad C_{1}(\alpha):=\frac{\sqrt{\pi} \Gamma\left(\frac{\alpha}{2}\right)}{\Gamma\left(\frac{\alpha+1}{2}\right)} .
$$

Thus

$$
\int_{\mathbb{R}}\left(\tilde{J}_{C_{1}, C_{2}}\right)^{-(1+\alpha) / 2} d t \leq 24 C_{1}(\alpha) m_{C_{1}, C_{2}}^{-\alpha / 2} C^{-\alpha} \frac{\sqrt{\tilde{K}_{C_{1}, C_{2}}(0)}}{I} .
$$

Using (4.12) and (4.29), the total change of direction is bounded from above by

$$
\begin{aligned}
\int_{\mathbb{R}} \frac{\left\|\dot{\tilde{q}}_{D} \wedge \ddot{\tilde{q}}_{D}\right\|}{\left\|\tilde{\tilde{q}}_{D}\right\|^{2}} d t & \leq \int_{\mathbb{R}} \frac{\left\|\ddot{\tilde{q}}_{D}\right\|}{\left\|\tilde{\tilde{q}}_{D}\right\|} d t \leq \frac{\sqrt{m_{C_{1}, C_{2}}}}{m_{D}} \int_{\mathbb{R}} \frac{\left\|\nabla \tilde{V}_{C_{1}, C_{2}}\right\|}{\sqrt{\tilde{K}_{C_{1}, C_{2}}}} d t \\
& \leq \frac{\sqrt{2 m_{C_{1}, C_{2}} / \tilde{K}_{C_{1}, C_{2}}(0)}}{m_{D}} \int_{\mathbb{R}}\left\|\nabla \tilde{V}_{C_{1}, C_{2}}\right\| d t \\
& \leq(1+c) \alpha \frac{I}{m_{D}} \sqrt{\frac{2 m_{C_{1}, C_{2}}}{\tilde{K}_{C_{1}, C_{2}}(0)}} \int_{\mathbb{R}}\left\|\tilde{q}_{D}\right\|^{-1-\alpha} d t \\
& \leq 2 \frac{I}{m_{D}} \sqrt{\frac{2 m_{C_{1}, C_{2}}}{\tilde{K}_{C_{1}, C_{2}}(0)}}\left(\frac{m_{D}}{\sqrt{2 m_{C_{1}, C_{2}}}}\right)^{\alpha+1} \int_{\mathbb{R}}\left(\tilde{J}_{C_{1}, C_{2}}\right)^{-(1+\alpha) / 2} d t \\
& \leq 48 C_{1}(\alpha)\left(\frac{m_{D}}{m_{C_{1}, C_{2}}}\right)^{\alpha} C^{-\alpha}
\end{aligned}
$$

for $C$ large. So as $C \nearrow \infty$, the total change of direction of $\tilde{q}_{C_{1}}^{\prime}=\tilde{q}_{D}^{\prime}$ goes to zero. (4.18) follows for cluster $C_{2}$, too, since $\frac{\tilde{q}_{C_{2}}^{\prime}(t)}{\left\|\tilde{q}_{C_{2}}^{\prime}(t)\right\|}=-\frac{\tilde{q}_{C_{1}}^{\prime}(t)}{\left\|\tilde{q}_{C_{1}}^{\prime}(t)\right\|}$ in the center of mass frame.

7. We next estimate the time evolutions of the energy terms, using the propagation estimate (4.27). We begin with $\tilde{H}_{D}^{I}$, see (4.9). A priori, the time derivative

$$
\frac{d}{d t} \tilde{H}_{D}^{I}=\left\langle\tilde{v}_{D}^{I}, \nabla_{q_{D}^{I}} V_{C_{1}, C_{2}}(\tilde{q})\right\rangle
$$

of the internal energy of cluster $D$ could be unbounded, since near-collisions of the particles 1 and 2 can lead to large relative velocities $\tilde{v}_{D}^{I}$. Therefore we apply partial integration when estimating the change of $\tilde{H}_{D}^{I}$.

$$
\begin{aligned}
\tilde{H}_{D}^{I}(t)-\tilde{H}_{D}^{I}(0) & =\int_{0}^{t} \frac{d}{d t} \tilde{H}_{D}^{I}(s) d s \\
& =\left.\left\langle\tilde{q}_{D}^{I}, \nabla_{q_{D}^{I}} V_{C_{1}, C_{2}}(\tilde{q})\right\rangle\right|_{0} ^{t}-\int_{0}^{t}\left\langle\tilde{q}_{D}^{I}, \frac{d}{d s} \nabla_{q_{D}^{I}} V_{C_{1}, C_{2}}(\tilde{q})\right\rangle d s .
\end{aligned}
$$

- By Statement 2, $\left\|\tilde{q}_{D}^{I}\right\| \ll\left\|\tilde{q}_{D}\right\|$ if $C$ is large. Then using (4.24) and the a priori inequality, the first term is bounded from above by $6 c_{1}^{-\alpha} C^{-\alpha} \tilde{K}_{C_{1}, C_{2}}(0)$. 
- Similarly we bound the integrand of the second term by

$$
\mid\left\langle\tilde{q}_{D}^{I}, \frac{d}{d s} \nabla_{q_{D}^{I}} V_{C_{1}, C_{2}}(\tilde{q})(s)\right| \leq 4 I\|\tilde{q}(s)\|^{-1-\alpha} \sqrt{\tilde{K}_{C_{1}, C_{2}}(0) / m_{C_{1}, C_{2}}} .
$$

Integration using (4.29) leads to the bound

$$
\left|\int_{0}^{t}\left\langle\tilde{q}_{D}^{I}, \frac{d}{d s} \nabla_{q_{D}^{I}} V_{C_{1}, C_{2}}(\tilde{q})\right\rangle d s\right| \leq 68 C_{1}(\alpha) C^{-\alpha} \tilde{K}_{C_{1}, C_{2}}(0) .
$$

So the variation of the internal energy of cluster $D$ is bounded by

$$
\left|\tilde{H}_{D}^{I}(t)-\tilde{H}_{D}^{I}(0)\right| \leq 74 C_{1}(\alpha) C^{-\alpha} \tilde{K}_{C_{1}, C_{2}}(0) .
$$

For $C$ large this is stronger than the a priori inequality (4.21).

The same bound applies to the variation of $\tilde{H}_{C_{1}, C_{2}}$, as the internal energy $H_{C_{1,2}}^{I}=H_{C_{1}, C_{2}}+H_{D}^{I}$ of $C_{1,2}$ is constant, see (4.8).

The estimate for the time evolution of $\tilde{V}_{C_{1}, C_{2}}$ follows directly from (4.25):

$$
\left|\tilde{V}_{C_{1}, C_{2}}(t)-\tilde{V}_{C_{1}, C_{2}}(0)\right| \leq 2 c_{2} C^{-\alpha} \tilde{K}_{C_{1}, C_{2}} .
$$

Def. (4.5) finally shows, using (4.31) and (4.32), that with $c_{3}:=2 c_{2}+74 C_{1}(\alpha)$

$$
\left|\tilde{K}_{C_{1}, C_{2}}(t)-\tilde{K}_{C_{1}, C_{2}}(0)\right| \leq c_{3} C^{-\alpha} \tilde{K}_{C_{1}, C_{2}} .
$$

For $C$ large this is stronger than the a priori inequality (4.19) and will show assertion (4.15).

8. The time evolution of the cluster angular momentum follows from

$$
\frac{d}{d t} \tilde{L}_{C_{1}, C_{2}}=-\frac{m_{D}}{m_{C_{1}, C_{2}}} \tilde{q}_{D} \wedge \nabla_{q_{D}^{I}} V_{C_{1}, C_{2}}(\tilde{q}) .
$$

So by an estimate similar to $(4.25)$

$$
\begin{aligned}
\left|\frac{d}{d t}\left\|\tilde{L}_{C_{1}, C_{2}}\right\|\right| & \leq\left\|\frac{d}{d t} \tilde{L}_{C_{1}, C_{2}}\right\| \leq 2 \alpha m_{D} m_{3} \frac{\left\|\tilde{q}_{D}^{I}\right\|}{\left(\frac{m_{D}}{m_{C_{1}, C_{2}}}\left\|\tilde{q}_{D}\right\|\right)^{1+\alpha}} \\
& =\alpha m_{D} m_{3}\left(\frac{m_{C_{1}, C_{2}}}{2}\right)^{(1+\alpha) / 2}\left\|\tilde{q}_{D}^{I}\right\|\left(\tilde{J}_{C_{1}, C_{2}}\right)^{-(1+\alpha) / 2}
\end{aligned}
$$

By (4.29) and Statement 2. on $\left\|\tilde{q}_{D}^{I}\right\|$

$$
\begin{aligned}
\left\|\tilde{L}_{C_{1}, C_{2}}(t)-\tilde{L}_{C_{1}, C_{2}}(0)\right\| & \leq 24 C_{1}(\alpha) \sqrt{m_{C_{1}, C_{2}}}\left\|\tilde{q}_{D}^{I}\right\| C^{-\alpha} \sqrt{\tilde{K}_{C_{1}, C_{2}}(0)} \\
& \leq C_{3} C^{-\alpha} \tilde{K}_{C_{1}, C_{2}}(0)^{-\frac{2-\alpha}{2 \alpha}} I^{1 / \alpha} \sqrt{m_{C_{1}, C_{2}}}
\end{aligned}
$$

with $C_{3}:=24 C_{1}(\alpha)\left(\frac{m_{D}^{I}}{m_{3}}\right)^{1 / \alpha}$. Comparing with (4.14), the relative variation of cluster angular momentum goes to zero as $C \nearrow \infty$, in accordance with a priori inequalities (4.20-4.21) and assertion (4.16). This finishes the proof. 
We now consider the influence of cluster $C_{3}$.

4.4 Lemma (Near-collision of $C_{1}$ and $C_{2}$, interacting with $C_{3}$ ) If $\left\|\tilde{q}_{C_{3}}(t)-\tilde{q}_{C_{1}}(t)\right\| \gg 1$ for $\left\{t \in \mathbb{R} \mid\left\|\tilde{q}_{D}(t)\right\| \leq 1\right\}$, the conclusions of Lemma 4.3 are still valid (with slightly worse constants), without its Assumption 3.

Proof: This follows from a perturbation argument concerning the forces, as in the proof of Lemma 4.3 we used the estimate (1.4) $\left(\left|\partial_{\beta} V_{i, j}(q)\right| \leq I\|q\|^{-\alpha-|\beta|}\right.$ for $\|q\|$ small, see also Remark 1.2). If $\left\|\tilde{q}_{C_{3}}(t)-\tilde{q}_{C_{1}}(t)\right\| \gg 1$ but $\left\|\tilde{q}_{D}(t)\right\| \leq 1$, then

So in particular, for $x \in \mathrm{NAV}_{E, 0}$ one has the reverse estimate for the relative angular momentum 4.3

$$
\left\|\tilde{L}_{C_{1}, C_{2}}(t)\right\| \leq 2 C \tilde{H}_{C_{1}, C_{2}}(0)^{-\frac{2-\alpha}{2 \alpha}} I^{1 / \alpha} \sqrt{m_{C_{1}, C_{2}}} \quad\left(t \in\left[0, t_{\max }\right]\right) .
$$

This will be used when we show that the orbit hits almost all Poincaré surfaces.

\subsection{Motion of the messenger cluster}

We consider the following setting: For $\left(C_{1}, C_{2}, C_{3}\right) \in \mathcal{T}$ the cluster center $q_{C_{2}}$ of $C_{2}$ moves in a time interval $\left(s_{1}, s_{3}\right)$ from a neighborhood of $q_{C_{1}}$ to the one of $q_{C_{3}}$. The respective neighborhoods are defined, using energy considerations.

For the total energy $E$ we can assume, using Lemma 4.1 (c), that the internal energy $\tilde{H}_{D}^{I}\left(s_{1}\right)$ of the nontrivial cluster $D$ is so negative that $|E| \ll-\tilde{H}_{D}^{I}\left(s_{1}\right)$. The times $s_{1}$ and $s_{3}$ are chosen so that the initial distances between $q_{C_{2}}$ and $q_{C_{i}}$ satisfy the inequality

$$
\left\|\tilde{q}_{C_{2}}-\tilde{q}_{C_{i}}\right\|\left(s_{i}\right) \geq C \max \left(\left(\tilde{K}_{C_{2}, C_{i}}\right)^{-1 / \varepsilon}\left(s_{i}\right), 1\right) \quad(i=1,3) .
$$

This condition means that in the time interval $\left(s_{1}, s_{3}\right)$, for $C$ large enough

1. one can apply the long range estimate (1.2), in particular $V_{i, j}(q)=\mathcal{O}\left(\|q\|^{-\varepsilon}\right)$, if $i$ and $j$ belong to different clusters;

2. thus the distances of the clusters are so large that the energies $\tilde{H}_{C_{2}, C_{i}}$ are mainly kinetic: $\left|\tilde{V}_{C_{2}, C_{i}}(t)\right| \ll \tilde{K}_{C_{2}, C_{i}}(t)$ for $t \in\left(s_{1}, s_{3}\right)$ and $C \gg 1$ in (4.36).

4.5 Lemma (Variation of angular momenta and directions) With (4.36),

1. the variations of the relative angular momenta are of order

$$
\left\|\tilde{L}_{C_{2}, C_{i}}\left(s_{1}\right)-\tilde{L}_{C_{2}, C_{i}}\left(s_{3}\right)\right\|=\mathcal{O}\left(\left(\tilde{K}_{C_{1}, C_{2}}\right)^{-1 / \alpha}\left(s_{1}\right)\right) \quad(i=1,3),
$$


2. the variations of the directions of the three clusters are of order

$$
\int_{s_{1}}^{s_{3}} \frac{\left\|\tilde{q}_{C_{2}, C_{i}}^{\prime} \wedge \tilde{q}_{C_{2}, C_{i}}^{\prime \prime}\right\|}{\left\|\tilde{q}_{C_{2}, C_{i}}^{\prime}\right\|^{2}} d t=\mathcal{O}\left(\left(\tilde{K}_{C_{1}, C_{2}}\right)^{-1 / \alpha}\left(s_{1}\right)\right) \quad(i=1,3),
$$

3. Consider the affine line $\left\{\tilde{q}_{C_{2}}\left(s_{2}\right)\right\}+\operatorname{span}\left(\tilde{p}_{C_{2}}\left(s_{2}\right)\right)$, with $s_{2}:=\frac{1}{2}\left(s_{1}+s_{3}\right)$. Then the maximal distance of $\tilde{q}_{C_{2}}(t)$ for $t \in\left(s_{1}, s_{3}\right)$ is of $\operatorname{order} \mathcal{O}\left(\left(\tilde{K}_{C_{1}, C_{2}}\right)^{-1 / \alpha}\left(s_{1}\right)\right)$. Similar statements hold for $C_{1}$ and $C_{3}$.

Thus all vanish in the limit $t \nearrow T(x)$.

4.6 Remark Note that for $i=3$, too the order depends on $\tilde{K}_{C_{1}, C_{2}}\left(s_{1}\right)$. This is because the kinetic energy comes into play through the estimate $\left\|\tilde{q}_{D}^{I}\right\|<$ $\left(\frac{2 I}{\tilde{K}_{C_{1}, C_{2}}}\right)^{1 / \alpha}$ in Lemma 4.3 for the size of the nontrivial cluster $D$. The difference is important, since unlike $\tilde{K}_{C_{2}, C_{3}}\left(s_{3}\right), \tilde{K}_{C_{1}, C_{2}}\left(s_{1}\right)$ is bounded below, by $\tilde{K}_{C_{2}}^{E}$ from Lemma 4.2 , since after collision $C_{1}$ and $C_{3}$ move in nearly opposite directions.

Proof: We proceed like in the proof of Lemma 4.3, with less details.

1. We use the inequalities $\tilde{J}_{C_{2}, C_{i}}(t) \geq \tilde{J}_{C_{2}, C_{i}}\left(s_{i}\right)+\frac{1}{4} \tilde{K}_{C_{2}, C_{i}}\left(s_{i}\right)\left(t-s_{i}\right)^{2}$, for $t \in\left(s_{1}, s_{2}\right)$, similar to (4.27). So

$$
\begin{aligned}
\int_{s_{1}}^{s_{3}}\left(\tilde{J}_{C_{2}, C_{i}}\right)^{-(1+\varepsilon) / 2} d t & \leq \frac{22^{(1+\varepsilon) / 2}}{\left(\tilde{K}_{C_{2}, C_{i}}\left(s_{i}\right)\right)^{1 / 2}} \int_{\left(\tilde{J}_{C_{2}, C_{i}}\left(s_{i}\right)\right)^{1 / 2}}^{\infty} t^{-1-\varepsilon} d t \\
& =\frac{22^{(1+\varepsilon) / 2}}{\varepsilon\left(\tilde{K}_{C_{2}, C_{i}}\left(s_{i}\right)\right)^{1 / 2}}\left(\tilde{J}_{C_{2}, C_{i}}\left(s_{i}\right)\right)^{-\varepsilon / 2},
\end{aligned}
$$

applying Cauchy-Schwarz. Thus by (4.36), the is integral bounded independent of $\tilde{K}_{C_{2}, C_{i}}\left(s_{i}\right)>0$, and of order $\mathcal{O}\left(\left(\tilde{K}_{C_{2}, C_{i}}\left(s_{i}\right)\right)^{-1 / 2}\right)$ for large relative kinetic cluster energies. With $\left|\frac{d}{d t}\left\|\tilde{L}_{C_{2}, C_{i}}\right\|\right| \leq c\left\|\tilde{q}_{D}^{I}\right\|\left(\tilde{J}_{C_{2}, C_{i}}\right)^{-(1+\alpha) / 2}$, see (4.34), and with (4.39), (4.37) follows, more precisely:

$$
\left.\left\|\tilde{L}_{C_{2}, C_{i}}\left(s_{1}\right)-\tilde{L}_{C_{2}, C_{i}}\left(s_{3}\right)\right\|=\mathcal{O}\left(\min \left(\tilde{K}_{C_{2}, C_{i}}\left(s_{i}\right)\right)^{-1 / 2}, 1\right) \tilde{K}_{C_{1}, C_{2}}\left(s_{1}\right)^{-\frac{1}{\alpha}}\right) .
$$

2. Unlike in (4.30), we use that the relative velocities of the clusters are nearly parallel to their relative positions (and thus their accelerations, as the $V_{i, j}$ are central). Thus we use a first order Taylor estimate for the integrand in (4.38). By (4.14) (remember that the NAV satisfy the converse inequality), initially

$$
\frac{\left\|\tilde{p}_{C_{2}, C_{i}}(t) \wedge \tilde{q}_{C_{2}, C_{i}}(t)\right\|}{\left\|\tilde{p}_{C_{2}, C_{i}}(t)\right\|}=\frac{\left\|\tilde{L}_{C_{2}, C_{i}}(t)\right\|}{\left\|\tilde{p}_{C_{2}, C_{i}}(t)\right\|}=\mathcal{O}\left(\tilde{K}_{C_{1}, C_{2}}(t)^{-\frac{1}{\alpha}}\right) \quad\left(t \in\left(s_{1}, s_{3}\right)\right),
$$

which is the same order as the size $\left\|\tilde{q}_{D}^{I}(t)\right\|$ of the nontrivial cluster. Therefore,

$$
\begin{aligned}
\frac{\left\|\tilde{q}_{C_{2}, C_{i}}^{\prime} \wedge \tilde{q}_{C_{2}, C_{i}}^{\prime \prime}\right\|}{\left\|\tilde{q}_{C_{2}, C_{i}}^{\prime}\right\|^{2}}(t) & =\mathcal{O}\left(\frac{\left\|D^{2} \tilde{V}_{C_{2}, C_{i}}(t)\right\|}{\left\|\tilde{q}_{C_{2}, C_{i}}^{\prime}(t)\right\|\left\|\tilde{q}_{C_{2}, C_{i}}(t)\right\|} \tilde{K}_{C_{1}, C_{2}}(t)^{-\frac{1}{\alpha}}\right) \\
& =\mathcal{O}\left(\left(\tilde{J}_{C_{2}, C_{i}}\right)^{-(3+\varepsilon) / 2}(t) \tilde{K}_{C_{1}, C_{2}}(t)^{-\frac{1}{\alpha}}\right) \quad\left(t \in\left(s_{1}, s_{3}\right)\right)
\end{aligned}
$$


Integration leads to the estimate

$$
\begin{aligned}
\int_{s_{1}}^{s_{3}} \frac{\left\|\tilde{q}_{C_{2}, C_{i}}^{\prime} \wedge \tilde{q}_{C_{2}, C_{i}}^{\prime \prime}\right\|}{\left\|\tilde{q}_{C_{2}, C_{i}}^{\prime}\right\|^{2}} d t & =\mathcal{O}\left(\int_{s_{i}}^{\infty} t^{-(3+\varepsilon) / 2} d t K_{C_{2}, C_{i}}\left(s_{i}\right)^{-(4+\varepsilon) / 2} \tilde{K}_{C_{1}, C_{2}}\left(s_{i}\right)^{-\frac{1}{\alpha}}\right) \\
& =\mathcal{O}\left(\tilde{K}_{C_{1}, C_{2}}\left(s_{1}\right)^{-\frac{1}{\alpha}}\right) .
\end{aligned}
$$

3. The third statement follows by double integration of the curvature 4.40 for the space curve $\tilde{q}_{C_{2}}$, starting at time $s_{2}$.

\section{Applicability of the Poincaré surface method}

To prove the main theorem (page 2), we show in Section 5.1 that NAV $\subseteq$ Wand, and in Section 5.2 that NAV $\subseteq$ Trans. Then the Poincaré surface method of Theorem 2.5 is applicable.

\subsection{NAV is wandering}

Proof of Theorem 2.8: The proof is based on the extension of von Zeipel's theorem in [Fl, Chapter 2.4], see also [Kn, Chapter 12.6]. As for lack of space we cannot fully reproduce that proof here, we indicate the main points.

It uses the Graf partition (5.3) of configuration space, originally devised in the context of quantum $n$-body scattering. This partition and its associated convex function (5.2) allow to focus attention on the motion of the cluster centers, instead of the more complicated cluster-internal motion, see [DG, Chapter 5].

For the partition lattice $\mathcal{P}(N)$ of $N=\{1, \ldots, n\}, \mathcal{C} \in \mathcal{P}(N)$, the projections $\Pi_{\mathcal{C}}^{E}, \Pi_{\mathcal{C}}^{I}: M \rightarrow M$ to the cluster-external/internal coordinates (see (2.6)), one sets

$$
J=J_{\mathcal{C}}^{E}+J_{\mathcal{C}}^{I} \quad \text { with } \quad J_{\mathcal{C}}^{E}:=J \circ \Pi_{\mathcal{C}}^{E} \quad \text { and } \quad J_{\mathcal{C}}^{I}:=J \circ \Pi_{\mathcal{C}}^{I},
$$

and, see Figure 5.1, for $\delta \in(0,1]$ sufficiently small

$$
J^{(\delta)}: M \rightarrow \mathbb{R} \quad, \quad J^{(\delta)}(q):=\max \left\{J_{\mathcal{C}}^{E}(q)+\delta^{|\mathcal{C}|} \mid \mathcal{C} \in \mathcal{P}(N)\right\} .
$$

The Graf partition of the configuration space $M$ is the family of subsets

$$
\Xi_{\mathcal{C}}^{(\delta)}:=\left\{q \in M \mid J_{C_{1}, C_{2}}(q)+\delta^{|\mathcal{C}|}=J^{(\delta)}(q)\right\} \quad(\mathcal{C} \in \mathcal{P}(N)) .
$$

$\Xi_{\mathcal{C}}^{(\delta)}$ should be compared with $\Xi_{\mathcal{C}}^{(0)}$ from (2.10).

Omitting the initial condition $x \in P$, there are piecewise constant mappings

$$
\mathcal{A}:(0, T(x)) \rightarrow \mathcal{P}(N) \quad \text { with } \quad \frac{\tilde{q}(t)}{t^{1-\varepsilon / 2}} \in \Xi_{\mathcal{A}(t)}^{(\delta)}
$$




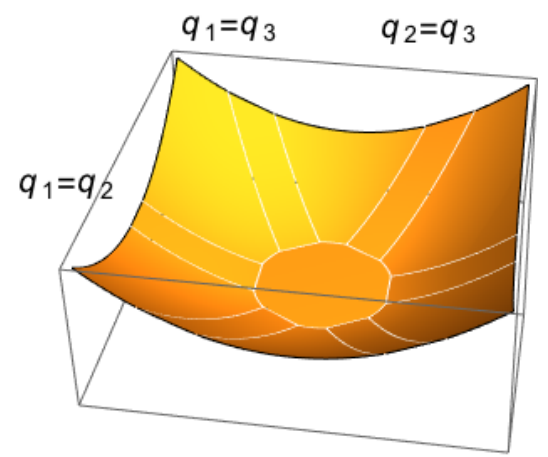

Figure 5.1: The function $J^{(\delta)}: M \rightarrow \mathbb{R}$ on the $n$-particle configuration space $M$, for $n=3$ particles in $d=1$ dimension and in the center of mass frame

(unique up to points on boundaries between the $\Xi_{\mathcal{A}(t)}^{(\delta)}$ ) and thus for $\tilde{Q}(t):=\frac{\tilde{q}(t)}{t}$ the continuous, piecewise differentiable approximant of $j$ (see (2.19)), $\tilde{j}^{(\delta)}(t):=\tilde{j}^{E}(t)+\delta^{|\mathcal{A}(t)|} t^{-\varepsilon} \quad$ with $\quad \tilde{j}^{E}(t):=J_{\mathcal{A}(t)}^{E}(\tilde{Q}(t))=\frac{1}{2}\left\langle\tilde{Q}_{\mathcal{A}(t)}^{E}, \tilde{Q}_{\mathcal{A}(t)}^{E}\right\rangle_{\mathcal{M}}$.

Omitting $\mathcal{A}$, the time derivative

$$
\frac{d}{d t} \tilde{j}^{E}=\frac{1}{2 t}\left\langle\tilde{Q}^{E}, \frac{d}{d t} \tilde{q}^{E}-\tilde{Q}^{E}\right\rangle_{\mathcal{M}}
$$

of the external part $\tilde{j}^{E}$ is written as a sum of a nonnegative function and a function on $\left(t_{0}, T(x)\right)$ whose modulus is smaller than $C t^{-1-\epsilon^{\prime}}$, for some $\epsilon^{\prime} \in$ $(0, \varepsilon)$ and for some $C>0$, only depending on $V$ and not on $x$. This shows existence of

$$
\lim _{t \nearrow T(x)} \tilde{j}^{E}(t) \in[0, \infty] .
$$

1. The remarks after (5.5) show that

$$
\left\|\frac{d}{d t} \tilde{q}^{E}(t)\right\|_{\mathcal{M}} \geq \frac{\left\langle\tilde{Q}^{E}(t), \frac{d}{d t} \tilde{q}^{E}(t)\right\rangle_{\mathcal{M}}}{\left\|\tilde{Q}^{E}(t)\right\|_{\mathcal{M}}} \geq\left\|\tilde{Q}^{E}(t)\right\|_{\mathcal{M}}-\mathcal{O}\left(t^{-\epsilon^{\prime}}\right) .
$$

For $x \in \mathrm{NAV}$ the term $\left\|\tilde{Q}^{E}(t)\right\|_{\mathcal{M}}=\left(2 \tilde{j}^{E}(t)\right)^{1 / 2}$ on the right side diverges: $\lim _{t \nearrow T(x)}\left\|\tilde{Q}^{E}(t)\right\|_{\mathcal{M}}=\infty$. Thus the external speed $\left\|\frac{d}{d t} \tilde{q}^{E}(t)\right\|_{\mathcal{M}}$ has the same limit. So kinetic energy goes to $\infty$, too and $\lim _{t \nearrow T(x)} \tilde{V}(t)=-\infty$.

2. As escape time $T: P \rightarrow(0, \infty]$ is lower semicontinuous, for any $\tau \in(0, T(x))$ there is a neighborhood $U$ of $x$ so that $\left.T\right|_{U} \geq \tau$. By shrinking $U$, if necessary, from continuity of the flow $\Phi$ and (5.6) we get for $y \in U$

$j^{E_{\circ}} \Phi_{\tau}(y) \geq j^{E_{\circ}} \Phi_{\tau}(x)-1$ and $j^{E_{\circ}} \Phi_{t}(y) \geq j^{E_{\circ}} \Phi_{\tau}(y)-C t^{-\epsilon^{\prime}}(t \in[\tau, T(y)))$.

As by increasing $\tau, j^{E} \circ \Phi_{\tau}(x)$ can be chosen arbitrarily large, $j^{+}$is lower semicontinuous at $x \in \mathrm{NAV}$. But as $j^{+}(x)=\infty, j^{+}$is continuous at $x$. 
3. As $J^{E}$ is bounded on $U$, this also shows that $x_{0}$ is wandering. As $j^{+}$is lower semicontinuous at NAV, for $k \in \mathbb{N}$ there are open neighborhoods $\mathrm{NAV}_{k} \supseteq$ NAV with $j^{+}(y)>k$ for $y \in \mathrm{NAV}_{k}$. So $\mathrm{NAV}=\bigcap_{k \in \mathbb{N}} \mathrm{NAV}_{k}$ is Borel.

\subsection{NAV is transitional}

For the admissible potentials $V$ of Definition 1.1, the pair potentials are central. So the total angular momentum $L$, see (2.13), is a constant of the motion.

This is also the case in the nondeterministic model of Sect. 3. But there, additionally the values of the cluster angular momenta are bounded by $|L|$, see (3.5).

Although that last property is not expected for the true dynamics and all times $t$, that is the case in the limit $t \nearrow T(x)$ :

\subsection{Lemma (Boundedness of the cluster angular momenta)}

For all $\ell>0$ and $x \in \mathrm{NAV}$ there is a minimal time $t_{0} \in(0, T(x))$ with

$$
\left\|\tilde{L}_{C}^{E}(t)\right\| \leq\|L(x)\|+\ell \quad \text { and } \quad\left\|\tilde{L}_{C}^{I}(t)\right\| \leq \ell \quad\left(t \in\left[t_{0}, T(x)\right), C \in \mathcal{A}(t)\right) .
$$

Concerning the $\tilde{L}_{C}^{E}$ estimate, the term $\ell>0$ is only needed if $\|L(x)\|=0$.

Proof: We have, see (2.14) and (2.15), the relation

$$
\tilde{L}(t)=\sum_{C \in \mathcal{A}(t)}\left(\tilde{L}_{C}^{E}(t)+\tilde{L}_{C}^{I}(t)\right) \quad(t \in[0, T(x))),
$$

and know from Section 2.5 that for $t \in\left[t_{0}, T(x)\right)$, the number of clusters is $|\mathcal{A}(t)| \in\{2,3\}$, changing infinitely often between the two cases.

1. For $t \in\left(t_{2 \ell}, t_{2 \ell+1}\right),|\mathcal{A}(t)|=3$ and there is a unique cluster $D(t) \in \mathcal{A}(t)$ of size $|D|=2$. By Lemma 4.1 (c) its internal energy $\tilde{H}_{D}^{I}=\tilde{K}_{D}^{I}+\tilde{V}_{D}^{I}$ goes to $-\infty$, and so does $\tilde{V}_{D}^{I} \leq \tilde{H}_{D}^{I}$. Using admissibility of $V$, $\left\|\tilde{q}_{D}^{I}\right\|=$ $\mathcal{O}\left(\left|\tilde{V}_{D}^{I}\right|^{-1 / \alpha}\right)=\mathcal{O}\left(\left|\tilde{H}_{D}^{I}\right|^{-1 / \alpha}\right)$, whereas $\left\|\tilde{p}_{D}^{I}\right\|=\mathcal{O}\left(\left|\tilde{H}_{D}^{I}\right|^{-1 / 2}\right)$. So internal angular momentum

$$
\left\|\tilde{L}_{D(t)}^{I}(t)\right\|=\mathcal{O}\left(\left(-\tilde{H}_{D(t)}^{I}(t)\right)^{-\frac{2-\alpha}{2 \alpha}}\right) \quad\left(t \in \cup_{\ell \in \mathbb{N}}\left(t_{2 \ell}, t_{2 \ell+1}\right)\right),
$$

going to zero as $t \nearrow T(x)$. $t$ belongs to an interval $\left(t_{2 \ell}, t_{2 \ell+1}\right)$ on which $\mathcal{A}$ is constant. Lemma 4.5 shows that on this interval the variation of the $\tilde{L}_{C}$ goes to zero, and at the end points, the relative angular momenta of the colliding clusters converge to zero as $t \nearrow T(x)$. In the kinematical formulae (3.13), $\left|L_{C}\right| \leq\left(1-\frac{m_{\min }}{M}\right)|L|$. As $\frac{m_{\min }}{M} \in(0,1)$, adding these dynamical terms finishes the proof for $|\mathcal{A}(t)|=3$ by increasing $t_{0}$ appropriately. 
2. For $t \in\left(t_{k}, t_{k+1}\right)$ with $k$ odd, that is $\left|\mathcal{A}\left(t_{k}^{+}\right)\right|=\left|\mathcal{A}\left(t_{k+1}^{-}\right)\right|=2$ clusters, one already knows from part 1 . of the proof that the three clusters present for $t \nearrow t_{k}$ respectively $t \searrow t_{k+1}$ meet the bounds of the lemma. A propagation estimate like (4.27) shows that within the interval $\left(t_{k}, t_{k+1}\right)$ the weak interaction between the two clusters does not suffice to violate these bounds.

So we can exhaust the set $\mathrm{NAV}_{E, 0}$ by first considering only the subset whose cluster angular momenta are bounded by $\mathfrak{L}>0$, show that this subset is of Liouville zero, and then let $\mathfrak{L} \nearrow \infty$.

Usually omitting the indices $E$ and $\mathfrak{L}$, for a given value $\mathfrak{L}>0$ we now define the family of Poincaré surfaces

$$
\mathcal{H}_{m} \equiv \mathcal{H}_{m, E, \mathfrak{L}}:=\bigcup_{\overline{\mathcal{C}} \in \mathcal{Q}} \mathcal{H}_{m}^{\overline{\mathcal{C}}} \subseteq \Sigma_{E, 0} \quad(m \in \mathbb{N}),
$$

with $\overline{\mathcal{C}}=\left(C_{1}, C_{2}, C_{3}\right)$ and $\left\{C_{1}, C_{2}, C_{3}\right\} \in \mathcal{P}_{3}(N)$, see page $11 . \mathcal{H}_{m}^{\overline{\mathcal{C}}}$ is defined so that the center of mass $q_{C_{2}}$ of the messenger cluster $C_{2}$ is in the hyperplane

$$
S_{m}\left(q_{C_{1}}\right):=\left\{r \in \mathbb{R}^{d} \mid\left\langle r, \frac{q_{C_{1}}}{\left\|q_{C_{1}}\right\|}\right\rangle=\left\|q_{C_{1}}\right\|-1 / m\right\}
$$

perpendicular to $q_{C_{1}}$, that has minimal distance $1 / m$ from $q_{C_{1}}$. The set $\mathcal{H}_{m}^{\bar{c}}$, defined in (5.11), will project to the hypersurface

$$
\mathcal{F}_{m}^{\overline{\mathcal{C}}}:=\left\{q \in M_{0} \mid\left\|q_{C_{1}}\right\| \geq 1, q_{C_{2}} \in S_{m}\left(q_{C_{1}}\right)\right\}
$$

of the $(3 d)$-dimensional center of mass configuration space

$$
M_{0}:=\left\{q \in M \mid q_{N}=0\right\} .
$$

We choose one of the two continuous unit normal vector fields

$$
N: \mathcal{F}_{m}^{\bar{c}} \rightarrow T_{\mathcal{F}_{m}^{\bar{c}}} M_{0} \quad, \quad N(q) \in \operatorname{span}\left(\nabla\left\langle r-q_{C_{1}}, \frac{q_{C_{1}}}{\left\|q_{C_{1}}\right\|}\right\rangle\right) .
$$

The normal component of $x \in \mathbb{R}^{d}$, w.r.t. $\operatorname{span}\left(q_{C_{1}}\right)$ equals

$$
x^{\perp}:=x-\frac{\left\langle x, q_{C_{1}}\right\rangle}{\left\|q_{C_{1}}\right\|^{2}} q_{C_{1}} .
$$

In (5.7) we now set (for a given parameter $\mathfrak{L}>0$ )

$$
\begin{array}{r}
\mathcal{H}_{m}^{\overline{\mathcal{C}}}:=\left\{(p, q) \in \Sigma_{E, 0} \mid\left\|p_{C_{2}}\right\| \geq m,\left\|q_{C_{1}}\right\| \geq 1, q_{C_{2}} \in S_{m}\left(q_{C_{1}}\right), p(N(q))<0,\right. \\
\left.\left\|q_{C_{2}}^{\perp}\right\|\left\|p_{C_{2}}\right\| \leq \mathfrak{L},\left\|p_{C_{2}}^{\perp}\right\|\left\|q_{C_{2}}\right\| \leq \mathfrak{L},\left\|p_{C_{1}}^{\perp}\right\|\left\|q_{C_{2}}\right\| \leq \mathfrak{L}\right\} .
\end{array}
$$

Physically, the condition $p(N(q))<0$ (with the pairing between $N(q) \in T_{q} M_{0}$ and $\left.p \in T_{q}^{*} M_{0}\right)$ means that the messenger cluster $C_{2}$ moves away from $C_{1}$, in the direction of $C_{3}$. Mathematically, it implies that $I_{m}$ is a volume form on $\mathcal{H}_{m}^{\overline{\mathcal{C}}}$. 
5.2 Lemma (Assumptions $\mathbf{A} 1$ and A2) For all $\mathfrak{L}>0$ the integrals

$$
I_{m}:=\sum_{\overline{\mathcal{C}} \in \mathcal{Q}} I_{m}^{\overline{\mathcal{C}}} \quad \text { with }^{3} \quad I_{m}^{\overline{\mathcal{C}}}:=\int_{\mathcal{H}_{m}^{\overline{\mathcal{C}}}} \mathcal{V}_{m}
$$

are finite, $\lim _{m \rightarrow \infty} I_{m}=0$, and the $(6 d-2)$-form $\mathcal{V}$ on $\Sigma_{E, 0}$ equals $\frac{\omega_{0}^{\wedge 3 d-1}}{(3 d-1) !}$.

The proof of that lemma will use a projection of the Poincare surface $\mathcal{H}_{m}^{\overline{\mathcal{C}}}$ to the cotangent bundle of $\mathcal{F}_{m}^{\overline{\mathcal{C}}}$ :

5.3 Lemma (Evaluation of the integrals) For $m \in \mathbb{N}$ large

$$
I_{m}^{\overline{\mathcal{C}}} \leq \int_{\mathcal{F}_{m}^{\overline{\mathcal{c}}}}\left(\int_{B_{r(q)}^{3 d-1}} \mathbb{1}_{y(q)}(p) d p\right) d \mathcal{F}(q),
$$

with $p \equiv\left(p_{C_{1}}, p_{C_{2}}^{\perp}, p_{D}^{I}\right), q \equiv\left(q_{C_{1}}, q_{C_{2}}^{\perp}, q_{D}^{I}\right)$, the Riemannian volume element $d \mathcal{F}$ on $\mathcal{F}_{m}^{\overline{\mathcal{C}}}$ (w.r.t. the norm $\|\cdot\|_{\mathcal{M}}$ on the tangent space, see (2.2)), the ball $B_{r(q)}^{3 d-1}$ of radius $r(q):=2(E-V(q))_{+}$(w.r.t. $\|\cdot\|_{\mathcal{M}^{-1}}$ on the cotangent space) and

$$
\begin{aligned}
y(q):=\left\{p \in B_{r(q)}^{3 d-1} \mid\right. & \left\|p_{C_{2}}\right\| \geq m, \\
& \left.\left\|q_{C_{2}}^{\perp}\right\|\left\|p_{C_{2}}\right\| \leq \mathfrak{L},\left\|p_{C_{2}}^{\perp}\right\|\left\|q_{C_{2}}\right\| \leq \mathfrak{L},\left\|p_{C_{1}}^{\perp}\right\|\left\|q_{C_{2}}\right\| \leq \mathfrak{L}\right\} .
\end{aligned}
$$

\subsection{Remark (Independent variables)}

Note that in the definition of $y(q),\left\|q_{C_{2}}\right\|$ and $\left\|p_{C_{2}}\right\|$ (unlike $\left\|q_{C_{2}}^{\perp}\right\|$ and $\left\|p_{C_{2}}^{\perp}\right\|$ ) appear as functions of the integration variables, given by (5.9), respectively by

$$
\frac{\left\|p_{C_{2}}\right\|^{2}}{2 m_{C_{2}}}=E-V(q)-\frac{\left\|p_{C_{1}}\right\|^{2}}{2 m_{C_{1}}}-\frac{\left\|p_{D}^{I}\right\|^{2}}{2 m_{D}^{I}} .
$$

Proof of Lemma 5.3:

$\mathcal{H}_{m}^{\overline{\mathcal{C}}}$ projects diffeomorphically to its image in $T^{*} \mathcal{F}_{m}^{\overline{\mathcal{C}}} \subseteq T^{*} M_{0}$, via

$$
n: \mathcal{H}_{m}^{\bar{c}} \rightarrow T^{*} \mathcal{F}_{m}^{\bar{C}} \quad, \quad(p, q) \mapsto\left(p-p(N(q), q) N^{b}(q)\right) .
$$

The cotangent bundle $T^{*} \mathcal{F}_{m}^{\overline{\mathcal{C}}}$ carries the canonical symplectic form $\omega_{\mathcal{F}}$. By Theorem $\mathrm{C}$ of [FK1], for the embedding $\imath: \mathcal{H}_{m}^{\overline{\mathcal{C}}} \rightarrow T^{*} M_{0}$ one has $\imath^{*} \omega_{0}=n^{*} \omega_{F}$. So

$$
\int_{\mathcal{H}_{m}^{\bar{c}}} \frac{\left(\imath^{*} \omega_{0}\right)^{\wedge 3 d-1}}{(3 d-1) !}=\int_{n\left(\mathcal{H}_{m}^{\bar{c}}\right)} \frac{\omega_{F}^{\wedge 3 d-1}}{(3 d-1) !},
$$

and we are left to determine the image $n\left(\mathcal{H}_{m}^{\overline{\mathcal{C}}}\right)$, that is, $p-p(N(q))$, see (5.10).

At the points where $q_{C_{2}}$ is linear dependent of $q_{C_{1}}, q_{C_{2}}^{\perp}=0$ in

$$
\nabla\left\langle q_{C_{2}}-q_{C_{1}}, \frac{q_{C_{1}}}{\left\|q_{C_{1}}\right\|}\right\rangle=\sum_{i=1}^{d}\left(m^{-1} \frac{q_{C_{1}, i}}{\left\|q_{C_{1}}\right\|}+\left\langle q_{C_{2}}^{\perp}, f_{i}\left(q_{C_{1}}\right)\right\rangle\right) \frac{\partial}{\partial q_{C_{1}, i}}+\frac{q_{C_{1}, i}}{\left\|q_{C_{1}}\right\|} \frac{\partial}{\partial q_{C_{2}, i}},
$$

\footnotetext{
${ }^{3}$ for the pull-back $\mathcal{V}_{m}=\iota_{m}^{*} \mathcal{V}$ of $\mathcal{V}=i_{X_{H}} \sigma_{E}$ w.r.t. the imbedding $\iota_{m}: \mathcal{H}_{m}^{\overline{\mathcal{C}}} \rightarrow \Sigma_{E, 0}$
} 
and thus its norm equals $\sqrt{1+1 / m^{2}}$. By comparison with the kinematical model, there is a constant such that after the $k$-th near-collision $\left\|p_{C_{1}}\right\| \geq c_{\bar{\mu}} \bar{\mu}^{k}$, for any $\bar{\mu} \in\left(1, \mu^{1 / 2}\right)$, with $\mu=1+\left(\frac{m_{\min }}{m_{\max }}\right)^{2}$, see (3.9). So by $\left\|q_{C_{2}}^{\perp}\right\|\left\|p_{C_{2}}\right\| \leq \mathfrak{L}$, $\left\|q_{C_{2}}^{\perp}\right\|$ is exponentially small in $k$. Dropping the normalizing factor $1 / \sqrt{1+1 / m^{2}}$ $\in(0,1)$, we can use (5.13) to estimate the integral.

\section{Proof of Lemma 5.2}

For an energy surface $\iota_{E}: \Sigma_{E}=H^{-1}(E) \rightarrow T^{*} M^{k}$, equality of $\mathcal{V}=\boldsymbol{i}_{X_{H}} \sigma_{E}$ for the Liouville volume form $\sigma_{E}$ on $\Sigma_{E}$ with $\frac{\left(\imath_{E}^{*} \omega_{0}\right)^{\wedge k-1}}{(k-1) !}$ was proven in [FK1, (6.1)]. Although (5.11) contains no explicit conditions concerning the nontrivial cluster $D \in\left\{C_{1}, C_{2}, C_{3}\right\}$, on $\mathcal{H}_{m}^{\overline{\mathcal{C}}}$ one has $\left\|p_{C_{2}}\right\| \geq m$ and thus $H_{D}^{I} \leq E-2 C m^{2}+$ $\mathcal{O}\left(m^{-\alpha}\right)$. Henceforth we only consider ${ }^{4} m>m_{0}$, with $m_{0} \in \mathbb{N}$ large enough such that $H_{D}^{I} \leq-C m^{2}$ (and that (5.12) applies). This entails $\left\|q_{D}^{I}\right\| \leq C m^{-2 / \alpha}$.

After performing the spherical integrations for $p_{C_{1}}^{\perp}, p_{C_{2}}^{\perp}$ and $p_{D}^{I}$, given the norms

$$
P \equiv\left(P_{C_{1}}, P_{C_{1}}^{\perp}, P_{C_{2}}^{\perp}, P_{D}^{I}\right):=\left(\left\|p_{C_{1}}\right\|,\left\|p_{C_{1}}^{\perp}\right\|,\left\|p_{C_{2}}^{\perp}\right\|,\left\|p_{D}^{I}\right\|\right)
$$

of the momenta, the r.h.s. of (5.12) equals

$$
c_{1} \int_{\mathcal{F}_{m}^{\overline{\mathcal{C}}}}\left(\int_{H_{r(q)}^{(4)} \cap Y(Q)}\left(P_{C_{1}}^{\perp}\right)^{d-2}\left(P_{C_{2}}^{\perp}\right)^{d-2}\left(P_{D}^{I}\right)^{d-1} d^{4} P\right) d \mathcal{F}(q)
$$

with

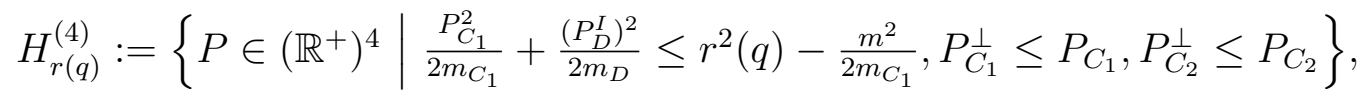

the Riemannian measure $s_{k}$ of the sphere $S^{k}, c_{1}:=s_{0}^{2} s_{d-2}^{2} s_{d-1}$ and, for

$$
\begin{gathered}
Q \equiv\left(Q_{C_{1}}, Q_{C_{1}}^{\perp}, Q_{C_{2}}^{\perp}, Q_{D}^{I}\right):=\left(\left\|q_{C_{1}}\right\|,\left\|q_{C_{1}}^{\perp}\right\|,\left\|q_{C_{2}}^{\perp}\right\|,\left\|q_{D}^{I}\right\|\right), \\
Y(Q):=\left\{P \in\left(\mathbb{R}^{+}\right)^{4} \mid Q_{C_{2}}^{\perp} \leq \mathfrak{L} / P_{C_{2}}, P_{C_{2}}^{\perp} \leq \mathfrak{L} / Q_{C_{2}}, P_{C_{1}}^{\perp} \leq \mathfrak{L} / Q_{C_{2}}\right\} .
\end{gathered}
$$

To perform the spherical integrations for the position $q$, we use that for $m$ large, on $\mathcal{H}_{m}$ the potential is dominated by $V(q) \leq-2 I\left(Q_{D}^{I}\right)^{-\alpha}$, since

- $\left\|q_{D}^{I}\right\|=\mathcal{O}\left(m^{-2 / \alpha}\right) \ll m^{-1}=\operatorname{dist}\left(S_{m}\left(q_{C_{1}}\right), q_{C_{1}}\right)$, and

- by the condition $q_{N}=0$, we have $q_{C_{3}}=-\frac{m_{C_{1}}+m_{C_{2}}\left(1-1 /\left(m\left\|q_{C_{1}}\right\|\right)\right)}{m_{C_{3}}} q_{C_{1}}-\frac{m_{C_{2}}}{m_{C_{3}}} q_{C_{2}}$ so that $\left\|q_{C_{3}}-q_{C_{i}}\right\| \geq \frac{m_{\min }}{m_{\max }}$ for $i=1,2$, using $\left\|q_{C_{1}}\right\| \geq 1$.

\footnotetext{
${ }^{4}$ in particular $m \geq 2$, so that $\left\|q_{C_{2}}\right\|$ is bounded away from zero, see (5.8).
} 
So, with $R(Q):=2\left(E+2 I\left(Q_{D}^{I}\right)^{-\alpha}\right)$ and $F_{m}:=\left\{Q \in(\mathbb{R}+)^{4} \mid Q_{C_{1}} \geq 1\right\}$, (5.14) is dominated for $d>2$ by

$$
\begin{aligned}
& c_{2} \int_{F_{m}}\left(\int_{H_{R(Q)}^{(4)} \cap Y(Q)}\left(P_{C_{1}}^{\perp}\right)^{d-2}\left(P_{C_{2}}^{\perp}\right)^{d-2}\left(P_{D}^{I}\right)^{d-1} d^{4} P\right) Q_{C_{1}}^{d-1}\left(Q_{C_{2}}^{\perp}\right)^{d-2}\left(Q_{D}^{I}\right)^{d-1} d^{4} Q \\
& \leq c_{3} \int_{1}^{\infty} \int_{0}^{m^{-\alpha}}\left(\int_{m}^{\infty} \int_{0}^{c\left(Q_{D}^{I}\right)^{-\alpha}}\left(P_{C_{2}}\right)^{1-d}\left(P_{D}^{I}\right)^{d-1} d P_{C_{2}} d P_{D}^{I}\right) Q_{C_{2}}^{2-2 d} Q_{C_{1}}^{d-1}\left(Q_{D}^{I}\right)^{d-1} d Q_{D}^{I} d Q_{C_{1}} \\
& \leq c_{4} m^{2-d} \int_{1}^{\infty} Q_{C_{1}}^{1-d} d Q_{C_{1}} \int_{0}^{m^{-\alpha}}\left(Q_{D}^{I}\right)^{d(1-\alpha / 2)-1} d Q_{D}^{I} \leq c_{5} m^{2-d-\alpha d(1-\alpha / 2)}
\end{aligned}
$$

Thus for $d \geq 3$ dimensions the integrals $I_{m}$ are finite, and $\lim _{m \rightarrow \infty} I_{m}=0$.

\subsection{Remark (The case of two dimensions)}

Note that although the integral $\int_{1}^{\infty} Q_{C_{1}}^{1-d} d Q_{C_{1}}$ diverges logarithmically for $d=2$, the power of $m$ in (5.15) is negative also in this case.

Probably one could extend our main result (page 2) to $d \geq 2$ by taking into account that the messenger particle has to return to $C_{1}^{\prime}$ after having experienced its near-collision with the cluster $C_{3}$. However, to prove that this effect leads to an additional negative power of $Q_{C_{1}}$ in (5.15) would require additional work. $\diamond$

5.6 Lemma If $x \in \mathrm{NAV}$, then for $\mathfrak{L}$ large enough in the definition (5.7) of Poincaré surfaces, the forward orbit hits almost all $\mathcal{H}_{m}$.

\section{Proof:}

We check the conditions in Def. (5.11) of the Poincare surfaces $\mathcal{H}_{m}^{\overline{\mathcal{C}}}, \overline{\mathcal{C}} \in \mathcal{Q}$ :

- $\left\|\tilde{p}_{C_{2}}(t)\right\| \geq m$ holds for $t<T(x)$ large by Lemma 4.2 , in combination with Theorem 2.8.

- $\left\|\tilde{q}_{C_{1}}\right\|(t) \geq 1$ follows for $t<T(x)$ large by von Zeipel's Theorem 2.6.

- $\tilde{q}_{C_{2}}(t) \in S_{m}\left(\tilde{q}_{C_{1}}(t)\right)$ with $\tilde{p}(N(\tilde{q}))<0$ holds for a sequence of times converging to $T(x)$, since, as shown in Section 2.5, the messenger cluster $C_{2}$ moves infinitely many times between disjoint neighborhoods of $\tilde{q}_{C_{1}}$ and $\tilde{q}_{C_{3}}$. As proven in Lemma 4.4 (see Assertion 2. of Lemma 4.3 for the (converse) estimate)), these neighborhoods are of radius $\mathcal{O}\left(\left(\tilde{K}_{C_{1}, C_{2}}\right)^{-1 / \alpha}\right)=\mathcal{O}\left(\left(\tilde{K}_{C_{2}}\right)^{-1 / \alpha}\right)$ which shrinks to zero as $t \nearrow T(x)$ by Lemma 4.2. So for any given $m \in \mathbb{N}$ they lie in different half-planes of $\mathbb{R}^{d}$, defined by $S_{m}$, see (5.8).

- The following three statements (5.16) all hold for parameter $\mathfrak{L} \geq 2\|\tilde{L}(0)\|$ and $t<T(x)$ large, since 
(a) By Lemma 4.5.3 the deviations of the trajectories $t \mapsto \tilde{q}_{C_{i}}$ of the cluster centers from motion on affine lines of $\mathbb{R}^{d}$ (whose definition is given in the lemma) becomes small.

(b) By Lemma $4.4 C_{2}$ has close encounters with $C_{1}$ and $C_{3}$, see above.

(c) At these Poincaré times $t$ the nontrivial cluster $D(t)$ has a size that goes to zero (see Assertion 2. of Lemma 4.3) and thus is compared to the minimal distance $\mathcal{O}(1 / m)$ of the cluster centers.

This is reflected in the angular momenta:

By Lemma 5.1 the cluster angular momenta $\tilde{L}_{C}^{E}(t)\|\leq\| L(x) \|+\ell$ for all $\ell>0$ and $t \in\left[t_{0}, T(x)\right), C \in \mathcal{A}(t)$ for $t_{0}(\ell) \in(0, T(x))$. Similarly, $\left\|\tilde{L}_{C}^{I}(t)\right\| \leq \ell$.

By Lemma 4.5.1, the variations of the relative angular momenta go to zero.

Thus we can compare with the straight line geometry of the kinematical model to see that for the above value of $\mathfrak{L}$ the violation of one of the three conditions

$$
\left\|q_{C_{2}}^{\perp}\right\|\left\|p_{C_{2}}\right\| \leq \mathfrak{L},\left\|p_{C_{2}}^{\perp}\right\|\left\|q_{C_{2}}\right\| \leq \mathfrak{L},\left\|p_{C_{1}}^{\perp}\right\|\left\|q_{C_{2}}\right\| \leq \mathfrak{L}
$$

would be in contradiction with $x \in \mathrm{NAV}$ and $t<T(x)$ large.

\section{References}

[De] Jan Dereziński: Large time behavior of classical $N$-body systems. Communications in Mathematical Physics 148, 503-520 (1992)

[DG] Jan Dereziński, Christian Gérard: Scattering Theory of Classical and Quantum N-Particle Systems. Texts and Monographs in Physics. Berlin: Springer, 1997

[FI] Stefan Fleischer: Improbability Results on Collision and Non-Collision Orbits in Multibody Systems via the Poincaré Surface Method. Dissertation. Available via OPUS FAU. FAU Erlangen-Nürnberg, 2018

[FK1] Stefan Fleischer, Andreas Knauf: Improbability of Wandering Orbits Passing Through a Sequence of Poincaré Surfaces of Decreasing Size. arXiv preprint, arXiv:1802.08566 (2018)

[FK2] Stefan Fleischer, Andreas Knauf: Improbability of Collisions in $n$-Body Systems. arXiv preprint, arXiv:1802.08564 (2018)

[FKM] Jacques Féjoz, Andreas Knauf, Richard Montgomery: Lagrangian Relations and Linear Point Billiards. Nonlinearity 30, 1326-1355 (2017) 
[Ge] Joseph Gerver: Noncollision Singularities: Do Four Bodies Suffice? Experimental Mathematics 12, 187-198 (2003)

[Kn] Andreas Knauf: Mathematical Physics: Classical Mechanics. Springer, 2018

[MS1] Christian Marchal, Donald Saari: On the Final Evolution of the $n$-Body Problem. Journal of Differential Equations 20, 150-186 (1976)

[Sa] Donald Saari: Expanding Gravitational Systems. Transactions of the AMS 156, 219-240 (1971)

[Sa1] Donald Saari: Improbability of Collisions in Newtonian Gravitational Systems. II. Transactions of the AMS 181, 351-368 (1973)

[Sa2] Donald Saari: A global existence theorem for the four body problem of Newtonian mechanics. J. Differential Equations 26, 80-111 (1977)

[SD] Donald Saari, Florin Diacu: Superhyperbolic expansion, noncollision singularities and symmetry configurations. Celestial Mechanics and Dynamical Astronomy 60, 91-98 (1994)

[SX] Donald Saari, Zhihong Xia: The existence of oscillatory and superhyperbolic motion in Newtonian systems. J. Differential Equations 82, 342-355 (1989)

[Sp] Hans Sperling: On the Real Singularities of the $n$-Body Problem. Journal für die reine und angewandte Mathematik 245, 15-40 (1970)

[Xi] Zhihong Xia: The existence of noncollision singularities in Newtonian systems. Annals of Mathematics 135, 411-468 (1992)

[Xu] Jinxin Xue: Noncollision singularities in a planar four-body problem. arXiv preprint, arXiv:1409.0048 (2014)

[Ze] Edvard Hugo von Zeipel: Sur les singularités du problème des $n$ corps. Arkiv f. Mat., Astr. och Fys. 4, Nr. 32, 4 S. (1908) 